\title{
Indiana Caregiving Survey Annotated Questionnaire
}

S1. Our study is interested in the opinions of people in certain age groups. Could you please tell me your age as of your last birthday?

\begin{tabular}{|l|c|c|c|c|}
\hline & \% ALL & \% SD5 & \% SD26 & \% SD39 \\
\hline $45-49$ & 13.5 & 12.0 & 12.4 & 14.9 \\
\hline $50-54$ & 14.2 & 14.0 & 12.8 & 15.9 \\
\hline $55-64$ & 30.7 & 32.0 & 28.0 & 30.2 \\
\hline $65+$ & 37.8 & 38.8 & 42.7 & 34.8 \\
\hline REF & 3.9 & 3.2 & 4.1 & 4.2 \\
\hline
\end{tabular}

S2. [If REFUSED in S1] I understand. Some people are not comfortable giving their age, but would you let me know which of the following age ranges you fall into?

\begin{tabular}{|l|c|c|c|c|}
\hline & \% ALL & \% SD5 & \% SD26 & \% SD39 \\
\hline $45-49$ & 21.6 & 41.1 & 23.2 & 23.4 \\
\hline $50-54$ & 14.1 & 0.0 & 29.7 & 14.8 \\
\hline $54-64$ & 21.4 & 20.5 & 15.5 & 22.6 \\
\hline $65+$ & 40.9 & 38.4 & 31.6 & 24.3 \\
\hline REF & 2.0 & 0.0 & 0.0 & 14.8 \\
\hline
\end{tabular}

S3. To ensure it is recorded accurately, could you please state your gender?

\begin{tabular}{|l|c|c|c|c|}
\hline & \% ALL & \% SD5 & \% SD26 & \% SD39 \\
\hline Male & 47.2 & 48.0 & 47.3 & 48.0 \\
\hline Female & 52.8 & 52.0 & 52.7 & 52.0 \\
\hline
\end{tabular}

S4. Are you registered to vote in Indiana?

\begin{tabular}{|l|c|c|c|c|}
\hline & \% ALL & \% SD5 & \% SD26 & \% SD39 \\
\hline Yes & 100.0 & 100.0 & 100.0 & 100.0 \\
\hline No & 0.0 & 0.0 & 0.0 & 0.0 \\
\hline
\end{tabular}

S5. What is your zip code?

\begin{tabular}{|l|c|c|c|c|}
\hline & \% ALL & \% SD5 & \% SD26 & \% SD39 \\
\hline Statewide & 100.0 & 0.0 & 0.0 & 0.0 \\
\hline District 5 & 12.1 & 100.0 & 0.0 & 0.0 \\
\hline District 26 & 12.0 & 0.0 & 100.0 & 0.0 \\
\hline District 39 & 11.9 & 0.0 & 0.0 & 100.0 \\
\hline
\end{tabular}




\section{MAIN QUESTIONNAIRE}

Q1. A family caregiver is someone who provides UNPAID care for an adult loved one who is ill, frail, elderly, or has a physical, mental, or emotional disability. This unpaid care may include assisting with personal needs like bathing and dressing, household chores, meals, shopping, transportation, or managing finances or medical care.

Are you currently a family caregiver providing UNPAID care to an adult loved one?

\begin{tabular}{|l|c|c|c|c|}
\hline & \% ALL & \% SD5 & \% SD26 & \% SD39 \\
\hline Yes & 18.1 & 13.1 & 23.7 & 14.6 \\
\hline No & 81.7 & 86.9 & 75.7 & 84.9 \\
\hline NS/REF & 0.3 & 0.0 & 0.6 & 0.5 \\
\hline
\end{tabular}

\section{QUESTIONS 2-17 ARE FOR CURRENT CAREGIVERS}

Q2. What is the age of the person you are providing care for?

\begin{tabular}{|l|c|c|c|c|}
\hline & \% ALL & \% SD5 & \% SD26 & \% SD39 \\
\hline Under 18 & 0.4 & 0.0 & 0.0 & 0.0 \\
\hline $18-49$ & 7.2 & 9.4 & 5.4 & 24.6 \\
\hline $50-59$ & 4.7 & 0.0 & 5.2 & 0.0 \\
\hline $60-64$ & 7.2 & 14.2 & 2.7 & 3.7 \\
\hline $65+$ & 79.5 & 76.4 & 82.7 & 71.7 \\
\hline NS/REF & 0.9 & 0.0 & 4.0 & 0.0 \\
\hline Mean Age & 75.4 & 75.5 & 76.6 & 74.1 \\
\hline
\end{tabular}

Q3. Still thinking about the person you are providing care to the most, which of the following activities are you providing? Are you ...?

\begin{tabular}{|c|c|c|c|}
\hline & \multicolumn{3}{|c|}{$\%$ ALL } \\
\hline & Yes & No & NS/REF \\
\hline a. Assisting with bathing or dressing & 41.0 & 57.7 & 1.3 \\
\hline b. Providing transportation to appointments & 81.1 & 18.9 & 0.0 \\
\hline c. Taking them shopping or shopping for them & 86.9 & 13.1 & 0.0 \\
\hline d. Helping them with meals & 75.6 & 24.0 & 0.4 \\
\hline e. Helping them to manage finances & 73.1 & 25.6 & 1.2 \\
\hline f. Aiding with household chores & 82.6 & 17.4 & 0.0 \\
\hline g. Providing social activities or companionship & 89.6 & 10.0 & 0.4 \\
\hline h. Overseeing their medication management & 68.2 & 31.4 & 0.4 \\
\hline i. Being responsible for other medical or nursing tasks & 68.6 & 29.8 & 1.5 \\
\hline
\end{tabular}




\begin{tabular}{|ll|c|c|c|}
\hline & \multicolumn{3}{|c|}{$\%$ SD5 } \\
\hline & Yes & No & NS/REF \\
\hline a. & Assisting with bathing or dressing & 60.0 & 34.9 & 5.1 \\
\hline b. & Providing transportation to appointments & 90.2 & 9.8 & 0.0 \\
\hline c. & Taking them shopping or shopping for them & 100.0 & 0.0 & 0.0 \\
\hline d. & Helping them with meals & 74.8 & 25.2 & 0.0 \\
\hline e. $\quad$ Helping them to manage finances & 95.2 & 4.8 & 0.0 \\
\hline f. & Aiding with household chores & 85.6 & 14.4 & 0.0 \\
\hline g. Providing social activities or companionship & 100.0 & 0.0 & 0.0 \\
\hline h. Overseeing their medication management & 70.4 & 29.6 & 0.0 \\
\hline i. & Being responsible for other medical or nursing tasks & 75.0 & 25.0 & 0.0 \\
\hline
\end{tabular}

\begin{tabular}{|ll|c|c|c|}
\hline & \multicolumn{3}{|c|}{$\%$ SD26 } \\
\hline & Yes & No & NS/REF \\
\hline a. & Assisting with bathing or dressing & 42.3 & 55.1 & 2.6 \\
\hline b. & Providing transportation to appointments & 96.0 & 4.0 & 0.0 \\
\hline c. & Taking them shopping or shopping for them & 88.0 & 12.0 & 0.0 \\
\hline d. Helping them with meals & 72.8 & 27.2 & 0.0 \\
\hline e. $\quad$ Helping them to manage finances & 72.6 & 24.8 & 2.6 \\
\hline f. & Aiding with household chores & 89.3 & 10.7 & 0.0 \\
\hline g. Providing social activities or companionship & 85.4 & 14.6 & 0.0 \\
\hline h. Overseeing their medication management & 77.6 & 22.4 & 0.0 \\
\hline i. & Being responsible for other medical or nursing tasks & 68.1 & 29.2 & 2.7 \\
\hline
\end{tabular}

\begin{tabular}{|ll|c|c|}
\hline & \multicolumn{3}{|c|}{$\%$ SD39 } \\
\hline & Yes & No & NS/REF \\
\hline a. Assisting with bathing or dressing & 48.0 & 48.3 & 3.7 \\
\hline b. Providing transportation to appointments & 84.3 & 15.7 & 0.0 \\
\hline c. $\quad$ Taking them shopping or shopping for them & 87.5 & 12.5 & 0.0 \\
\hline d. Helping them with meals & 84.9 & 15.1 & 0.0 \\
\hline e. Helping them to manage finances & 83.8 & 16.2 & 0.0 \\
\hline f. Aiding with household chores & 84.9 & 15.1 & 0.0 \\
\hline g. Providing social activities or companionship & 83.2 & 16.8 & 0.0 \\
\hline h. Overseeing their medication management & 65.0 & 35.0 & 0.0 \\
\hline i. $\quad$ Being responsible for other medical or nursing tasks & 84.5 & 15.5 & 0.0 \\
\hline
\end{tabular}

Q3b1. Do you drive your loved one to medical appointments?

\begin{tabular}{|l|c|c|c|c|}
\hline & \% ALL & \% SD5 & \% SD26 & \% SD39 \\
\hline Yes & 96.9 & 94.7 & 97.3 & 100.0 \\
\hline No & 3.1 & 5.3 & 2.7 & 0.0 \\
\hline NS/REF & 0.0 & 0.0 & 0.0 & 0.0 \\
\hline
\end{tabular}


Q4. How often do you [a-i]:

\begin{tabular}{|c|c|c|c|c|}
\hline & \multicolumn{4}{|c|}{$\%$ ALL } \\
\hline & Daily & Weekly & Monthly & NS/REF \\
\hline a. Assist with bathing or dressing & 54.6 & 27.5 & 13.8 & 4.1 \\
\hline b. Provide transportation to appointments & 12.7 & 39.0 & 41.8 & 6.5 \\
\hline c. Take them shopping or shop for them & 11.4 & 62.7 & 21.9 & 3.9 \\
\hline d. Help them with meals & 63.2 & 22.9 & 9.4 & 4.5 \\
\hline e. Help them to manage finances & 45.3 & 25.1 & 29.0 & 0.6 \\
\hline f. Aid with household chores & 52.9 & 33.4 & 11.0 & 2.6 \\
\hline g. Provide social activities or companionship & 63.2 & 28.7 & 6.3 & 1.9 \\
\hline h. Oversee their medication management & 62.0 & 20.5 & 13.1 & 4.4 \\
\hline $\begin{array}{l}\text { i. Be responsible for other medical or nursing } \\
\text { tasks }\end{array}$ & 42.2 & 29.7 & 21.0 & 7.1 \\
\hline
\end{tabular}

\begin{tabular}{|c|c|c|c|c|}
\hline & \multicolumn{4}{|c|}{$\%$ SD5 } \\
\hline & Daily & Weekly & Monthly & NS/REF \\
\hline a. Assist with bathing or dressing & 84.2 & 7.8 & 0.0 & 7.9 \\
\hline b. Provide transportation to appointments & 17.0 & 34.8 & 42.4 & 5.8 \\
\hline c. Take them shopping or shop for them & 14.5 & 51.1 & 34.4 & 0.0 \\
\hline d. Help them with meals & 74.2 & 6.4 & 6.3 & 13.2 \\
\hline e. Help them to manage finances & 48.1 & 25.9 & 26.0 & 0.0 \\
\hline f. Aid with household chores & 70.0 & 30.0 & 0.0 & 0.0 \\
\hline g. Provide social activities or companionship & 79.9 & 15.3 & 0.0 & 4.8 \\
\hline h. Oversee their medication management & 85.5 & 14.5 & 0.0 & 0.0 \\
\hline $\begin{array}{l}\text { i. Be responsible for other medical or nursing } \\
\text { tasks }\end{array}$ & 53.8 & 46.2 & 0.0 & 0.0 \\
\hline
\end{tabular}

\begin{tabular}{|c|c|c|c|c|}
\hline & \multicolumn{4}{|c|}{$\%$ SD26 } \\
\hline & Daily & Weekly & Monthly & NS/REF \\
\hline a. Assist with bathing or dressing & 57.5 & 30.0 & 12.5 & 0.0 \\
\hline b. Provide transportation to appointments & 17.0 & 34.8 & 42.4 & 5.8 \\
\hline c. Take them shopping or shop for them & 18.3 & 54.7 & 17.8 & 9.2 \\
\hline d. Help them with meals & 61.6 & 31.3 & 7.1 & 0.0 \\
\hline e. Help them to manage finances & 65.5 & 20.0 & 14.5 & 0.0 \\
\hline f. Aid with household chores & 45.0 & 37.4 & 17.6 & 0.0 \\
\hline g. Provide social activities or companionship & 56.2 & 34.5 & 6.2 & 3.1 \\
\hline h. Oversee their medication management & 52.1 & 30.8 & 17.0 & 0.0 \\
\hline $\begin{array}{l}\text { i. Be responsible for other medical or nursing } \\
\text { tasks }\end{array}$ & 39.8 & 39.0 & 13.8 & 7.5 \\
\hline
\end{tabular}




\begin{tabular}{|ll|c|c|c|c|}
\hline & \multicolumn{4}{|c|}{$\%$ SD39 } \\
\hline & Daily & Weekly & Monthly & NS/REF \\
\hline a. & Assist with bathing or dressing & 42.4 & 32.0 & 25.6 & 0.0 \\
\hline b. & Provide transportation to appointments & 4.3 & 41.1 & 41.2 & 13.3 \\
\hline c. & Take them shopping or shop for them & 10.1 & 54.1 & 27.4 & 8.4 \\
\hline d. & Help them with meals & 51.4 & 24.9 & 14.8 & 8.9 \\
\hline e. & Help them to manage finances & 56.7 & 18.0 & 25.2 & 0.0 \\
\hline f. & Aid with household chores & 51.4 & 19.4 & 29.2 & 0.0 \\
\hline g. & Provide social activities or companionship & 67.1 & 22.6 & 10.4 & 0.0 \\
\hline h. & Oversee their medication management & 74.7 & 19.3 & 6.0 & 0.0 \\
\hline i. & $\begin{array}{l}\text { Be responsible for other medical or nursing } \\
\text { tasks }\end{array}$ & 37.4 & 33.0 & 29.6 & 0.0 \\
\hline
\end{tabular}

Q5. Thinking about the person you provide care to, about how long would it take you, in minutes, to this person to:

\begin{tabular}{|l|c|c|c|}
\hline & \multicolumn{3}{|c|}{ \% ALL } \\
\hline & The nearest hospital & $\begin{array}{c}\text { A doctor's office for } \\
\text { primary care }\end{array}$ & $\begin{array}{c}\text { A doctor's office for } \\
\text { specialty care }\end{array}$ \\
\hline Less than 5 minutes & 4.3 & 4.0 & 1.7 \\
\hline 5-10 minutes & 21.3 & 18.9 & 10.4 \\
\hline 11-15 minutes & 23.2 & 15.0 & 14.8 \\
\hline 16-30 minutes & 33.5 & 40.2 & 35.0 \\
\hline 31-45 minutes & 10.6 & 6.6 & 11.7 \\
\hline 46 minutes to an hour & 2.6 & 4.9 & 11.0 \\
\hline More than 1 hour & 3.3 & 7.4 & 11.7 \\
\hline NS/REF & 1.3 & 3.0 & 3.7 \\
\hline
\end{tabular}

\begin{tabular}{|l|c|c|c|}
\hline & \multicolumn{3}{|c|}{ \% SD5 } \\
\hline & The nearest hospital & $\begin{array}{c}\text { A doctor's office for } \\
\text { primary care }\end{array}$ & $\begin{array}{c}\text { A doctor's office for } \\
\text { specialty care }\end{array}$ \\
\hline Less than 5 minutes & 0.0 & 0.0 & 0.0 \\
\hline 5-10 minutes & 10.2 & 14.9 & 9.8 \\
\hline 11-15 minutes & 5.7 & 4.9 & 0.0 \\
\hline 16-30 minutes & 59.9 & 41.0 & 29.1 \\
\hline 31-45 minutes & 14.4 & 4.8 & 27.0 \\
\hline 46 minutes to an hour & 5.1 & 19.6 & 19.7 \\
\hline More than 1 hour & 4.8 & 9.7 & 14.4 \\
\hline NS/REF & 0.0 & 5.1 & 0.0 \\
\hline
\end{tabular}




\begin{tabular}{|l|c|c|c|}
\hline & \multicolumn{3}{|c|}{ \% SD26 } \\
\hline The nearest hospital & $\begin{array}{c}\text { A doctor's office for } \\
\text { primary care }\end{array}$ & $\begin{array}{c}\text { A doctor's office for } \\
\text { specialty care }\end{array}$ \\
\hline Less than 5 minutes & 7.4 & 10.0 & 0.0 \\
\hline $5-10$ minutes & 27.3 & 10.8 & 9.4 \\
\hline 11-15 minutes & 15.8 & 7.8 & 5.1 \\
\hline 16-30 minutes & 38.6 & 55.2 & 44.6 \\
\hline 31-45 minutes & 10.9 & 8.2 & 14.7 \\
\hline 46 minutes to an hour & 0.0 & 0.0 & 15.5 \\
\hline More than 1 hour & 0.0 & 5.4 & 8.0 \\
\hline NS/REF & 0.0 & 2.7 & 2.7 \\
\hline
\end{tabular}

\begin{tabular}{|l|c|c|c|}
\hline & \multicolumn{3}{|c|}{ \% SD39 } \\
\hline The nearest hospital & $\begin{array}{c}\text { A doctor's office for } \\
\text { primary care }\end{array}$ & $\begin{array}{c}\text { A doctor's office for } \\
\text { specialty care }\end{array}$ \\
\hline 5-10 minutes & $0 . .0$ & 0.0 & 3.7 \\
\hline 11-15 minutes & 48.1 & 27.0 & 7.6 \\
\hline 16-30 minutes & 27.9 & 16.2 & 19.8 \\
\hline 31-45 minutes & 11.5 & 40.4 & 32.6 \\
\hline 46 minutes to an hour & 5.0 & 0.0 & 0.0 \\
\hline More than 1 hour & 0.0 & 0.0 & 7.8 \\
\hline NS/REF & 0.0 & 12.5 & 12.8 \\
\hline
\end{tabular}

Q6. Thinking about the person you provide care to, and the county they live in, do you need to travel out of their county to get to ...?

\begin{tabular}{|c|c|c|c|}
\hline & \multicolumn{3}{|c|}{$\%$ ALL } \\
\hline & Yes & No & NS/REF \\
\hline a. The nearest hospital & 22.9 & 77.1 & 0.0 \\
\hline b. A doctor's office for primary care & 21.9 & 77.2 & 0.9 \\
\hline $\begin{array}{l}\text { c. A doctor's office for specialty care, like a cardiologist, } \\
\text { neurologist, or other specialist }\end{array}$ & 39.6 & 60.0 & 0.4 \\
\hline
\end{tabular}

\begin{tabular}{|l|c|c|c|}
\hline & \multicolumn{3}{|c|}{$\%$ SD5 } \\
\hline & Yes & No & NS/REF \\
\hline a. The nearest hospital & 55.8 & 44.2 & 0.0 \\
\hline b. A doctor's office for primary care & 45.9 & 49.0 & 5.1 \\
\hline $\begin{array}{l}\text { c. A doctor's office for specialty care, like a cardiologist, } \\
\text { neurologist, or other specialist }\end{array}$ & 71.1 & 28.9 & 0.0 \\
\hline
\end{tabular}

\begin{tabular}{|l|c|c|c|}
\hline & \multicolumn{3}{|c|}{ \% SD26 } \\
\hline & Yes & No & NS/REF \\
\hline a. The nearest hospital & 7.9 & 92.1 & 0.0 \\
\hline b. A doctor's office for primary care & 10.6 & 86.7 & 2.7 \\
\hline $\begin{array}{l}\text { c. A doctor's office for specialty care, like a cardiologist, } \\
\text { neurologist, or other specialist }\end{array}$ & 42.1 & 57.9 & 0.0 \\
\hline
\end{tabular}




\begin{tabular}{|l|c|c|c|}
\hline & \multicolumn{3}{|c|}{ \% SD39 } \\
\hline & Yes & No & NS/REF \\
\hline a. The nearest hospital & 7.8 & 92.2 & 0.0 \\
\hline b. A doctor's office for primary care & 16.7 & 83.3 & 0.0 \\
\hline $\begin{array}{l}\text { c. A doctor's office for specialty care, like a cardiologist, } \\
\text { neurologist, or other specialist }\end{array}$ & 24.3 & 75.7 & 0.0 \\
\hline
\end{tabular}

Q7. Have you ever been employed either full- or part-time while providing care to your adult loved one?

\begin{tabular}{|l|c|c|c|c|}
\hline & \% ALL & \% SD5 & \% SD26 & \% SD39 \\
\hline Yes & 61.2 & 66.3 & 54.6 & 68.0 \\
\hline No & 38.8 & 33.7 & 45.4 & 32.0 \\
\hline DK/REF & 0.0 & 0.0 & 0.0 & 0.0 \\
\hline
\end{tabular}

Q8. Now thinking again about the adult loved one you are currently providing care to, did you ever have to ...?

\begin{tabular}{|l|c|c|c|}
\hline & \multicolumn{3}{|c|}{ \% ALL } \\
\hline $\begin{array}{l}\text { a. Go into work early or late, or take time off to provide care } \\
\text { for a loved one }\end{array}$ & 76.5 & 23.5 & 0.0 \\
\hline $\begin{array}{l}\text { b. Take a leave of absence from your job to provide care for } \\
\text { a loved one }\end{array}$ & 33.2 & 65.7 & 1.1 \\
\hline $\begin{array}{l}\text { c. Go from working full-time to part-time to provide care for } \\
\text { a loved one }\end{array}$ & 18.6 & 80.7 & 0.7 \\
\hline d. Give up working entirely to provide care for a loved one & 20.9 & 79.1 & 0.0 \\
\hline
\end{tabular}

\begin{tabular}{|l|c|c|c|}
\hline & \multicolumn{3}{|c|}{$\%$ SD5 } \\
\hline $\begin{array}{l}\text { a. Go into work early or late, or take time off to provide care } \\
\text { for a loved one }\end{array}$ & 85.7 & 14.3 & 0.0 \\
\hline \begin{tabular}{l} 
b. $\begin{array}{l}\text { Take a leave of absence from your job to provide care for } \\
\text { a loved one }\end{array}$ \\
\hline $\begin{array}{l}\text { c. Go from working full-time to part-time to provide care for } \\
\text { a loved one }\end{array}$
\end{tabular} & 24.5 & 70.3 & 0.0 \\
\hline d. Give up working entirely to provide care for a loved one & 31.8 & 68.2 & 0.0 \\
\hline
\end{tabular}

\begin{tabular}{|l|c|c|c|}
\hline & \multicolumn{3}{|c|}{ \% SD26 } \\
\hline $\begin{array}{l}\text { a. Go into work early or late, or take time off to provide care } \\
\text { for a loved one }\end{array}$ & 65.6 & 34.4 & 0.0 \\
\hline $\begin{array}{l}\text { b. Take a leave of absence from your job to provide care for } \\
\text { a loved one }\end{array}$ & 28.4 & 71.6 & 0.0 \\
\hline \begin{tabular}{l} 
c. $\quad \begin{array}{l}\text { Go from working full-time to part-time to provide care for } \\
\text { a loved one }\end{array}$ \\
\hline d. Give up working entirely to provide care for a loved one
\end{tabular} & 23.4 & 76.6 & 0.0 \\
\hline
\end{tabular}




\begin{tabular}{|l|c|c|c|}
\hline & \multicolumn{3}{|c|}{$\%$ SD39 } \\
\hline $\begin{array}{l}\text { a. Go into work early or late, or take time off to provide care } \\
\text { for a loved one }\end{array}$ & 94.6 & 5.4 & 0.0 \\
\hline $\begin{array}{l}\text { b. Take a leave of absence from your job to provide care for } \\
\text { a loved one }\end{array}$ & 28.8 & 59.6 & 11.5 \\
\hline $\begin{array}{l}\text { c. Go from working full-time to part-time to provide care for } \\
\text { a loved one }\end{array}$ & 16.2 & 78.4 & 5.4 \\
\hline d. Give up working entirely to provide care for a loved one & 21.9 & 78.1 & 0.0 \\
\hline
\end{tabular}

Q9. [IF Q8A=YES ASK] You mentioned that you had to go into work early or late, or take time off to provide care to an adult loved one. How often would you say you had to take time away from work or adjust your work schedule to provide care for someone? Would you say ...?

\begin{tabular}{|l|c|c|c|c|}
\hline & \% ALL & \% SD5 & \% SD26 & \% SD39 \\
\hline More than once per week & 11.0 & 9.0 & 7.5 & 13.8 \\
\hline Once per week & 12.7 & 19.9 & 20.3 & 17.5 \\
\hline $\begin{array}{l}\text { Several times per month but less often } \\
\text { than once per week }\end{array}$ & 22.0 & 35.2 & 34.9 & 0.0 \\
\hline Once per month & 26.0 & 27.6 & 7.6 & 37.5 \\
\hline Less than once per month & 24.1 & 8.3 & 29.6 & 19.1 \\
\hline DK/REF & 4.2 & 0.0 & 0.0 & 12.2 \\
\hline
\end{tabular}

Q10. Now thinking again about the adult loved one you are currently providing care to, did you ever have to ...?

\begin{tabular}{|l|c|c|c|}
\hline & \multicolumn{3}{|c|}{ \% ALL } \\
\hline & Yes & No & NS/REF \\
\hline a. Use your own money to help provide care & 72.1 & 27.1 & 0.8 \\
\hline b. Make changes to your home for the loved one in your care & 41.2 & 58.5 & 0.0 \\
\hline c. Move into another home to accommodate the loved one in & 15.6 & 84.4 & 0.0 \\
\hline
\end{tabular}

\begin{tabular}{|l|c|c|c|}
\hline & \multicolumn{3}{|c|}{$\%$ SD5 } \\
\hline & Yes & No & NS/REF \\
\hline a. Use your own money to help provide care & 79.6 & 20.4 & 0.0 \\
\hline b. Make changes to your home for the loved one in your care & 45.2 & 54.8 & 0.0 \\
\hline $\begin{array}{l}\text { c. } \text { Move into another home to accommodate the loved one in } \\
\text { your care }\end{array}$ & 9.8 & 90.2 & 0.0 \\
\hline
\end{tabular}

\begin{tabular}{|l|c|c|c|}
\hline & \multicolumn{3}{|c|}{$\%$ SD26 } \\
\hline & Yes & No & NS/REF \\
\hline a. Use your own money to help provide care & 61.5 & 38.5 & 0.0 \\
\hline b. Make changes to your home for the loved one in your care & 29.7 & 70.3 & 0.0 \\
\hline $\begin{array}{l}\text { c. Move into another home to accommodate the loved one in } \\
\text { your care }\end{array}$ & 16.1 & 83.9 & 0.0 \\
\hline
\end{tabular}




\begin{tabular}{|l|c|c|c|}
\hline & \multicolumn{3}{|c|}{ \% SD39 } \\
\hline a. Use your own money to help provide care & Yes & No & NS/REF \\
\hline b. Make changes to your home for the loved one in your care & 71.8 & 24.3 & 3.9 \\
\hline c. Move into another home to accommodate the loved one in & 15.1 & 64.8 & 0.0 \\
\hline & & 84.9 & 0.0 \\
\hline
\end{tabular}

Q11. As a caregiver do you ever ...?

\begin{tabular}{|ll|c|c|c|}
\hline & \multicolumn{3}{|c|}{$\%$ ALL } \\
\hline $\begin{array}{l}\text { a. } \\
\text { Feel stressed emotionally due to your caregiving } \\
\text { responsibilities }\end{array}$ & Yes & No & NS/REF \\
\hline b. & Feel stressed in trying to balance your job and family & 33.8 & 1.3 \\
\hline c. & Find it difficult to maintain a healthy diet & 61.1 & 37.5 & 1.4 \\
\hline d. & Find it difficult to get enough rest & 32.5 & 66.2 & 1.3 \\
\hline e. & Find it difficult to exercise regularly & 56.7 & 43.3 & 0.0 \\
\hline f. $\quad$ Find it difficult to take care of your household & 40.1 & 59.1 & 0.9 \\
\hline responsibilities & 42.4 & 55.4 & 2.1 \\
\hline g. Experience problems with your health & 31.1 & 68.5 & 0.4 \\
\hline h. Find it difficult to make time to visit your doctor & 24.9 & 74.3 & 0.8 \\
\hline i. & Feel strained financially due to your caregiving & 31.1 & 67.6 & 1.2 \\
\hline & responsibilities & & & \\
\hline
\end{tabular}

\begin{tabular}{|c|c|c|c|}
\hline & \multicolumn{3}{|c|}{$\%$ SD5 } \\
\hline & Yes & No & NS/REF \\
\hline $\begin{array}{l}\text { a. Feel stressed emotionally due to your caregiving } \\
\text { responsibilities }\end{array}$ & 74.9 & 25.1 & 0.0 \\
\hline b. Feel stressed in trying to balance your job and family & 61.1 & 38.9 & 0.0 \\
\hline c. Find it difficult to maintain a healthy diet & 38.8 & 61.2 & 0.0 \\
\hline d. Find it difficult to get enough rest & 60.1 & 39.9 & 0.0 \\
\hline e. Find it difficult to exercise regularly & 45.6 & 54.4 & 0.0 \\
\hline $\begin{array}{l}\text { f. Find it difficult to take care of your household } \\
\text { responsibilities }\end{array}$ & 45.0 & 55.0 & 0.0 \\
\hline g. Experience problems with your health & 45.0 & 55.0 & 0.0 \\
\hline h. Find it difficult to make time to visit your doctor & 35.8 & 64.2 & 0.0 \\
\hline $\begin{array}{l}\text { i. Feel strained financially due to your caregiving } \\
\text { responsibilities }\end{array}$ & 40.2 & 55.1 & 4.8 \\
\hline
\end{tabular}




\begin{tabular}{|ll|c|c|c|}
\hline & \multicolumn{3}{|c|}{$\%$ SD26 } \\
\hline a. & Feel stressed emotionally due to your caregiving & Yes & No & NS/REF \\
\hline responsibilities & 74.2 & 23.1 & 2.7 \\
\hline b. & Feel stressed in trying to balance your job and family & 66.2 & 33.8 & 0.0 \\
\hline c. & Find it difficult to maintain a healthy diet & 31.5 & 65.8 & 2.7 \\
\hline d. Find it difficult to get enough rest & 63.1 & 36.9 & 0.0 \\
\hline e. & Find it difficult to exercise regularly & 36.8 & 60.5 & 2.7 \\
\hline f. $\begin{array}{l}\text { Find it difficult to take care of your household } \\
\text { responsibilities }\end{array}$ & 51.0 & 46.2 & 2.7 \\
\hline g. & & & \\
\hline hexperience problems with your health & Find it difficult to make time to visit your doctor & 26.5 & 73.5 & 0.0 \\
\hline i. $\begin{array}{l}\text { Feel strained financially due to your caregiving } \\
\text { responsibilities }\end{array}$ & 28.8 & 68.5 & 2.7 \\
\hline
\end{tabular}

\begin{tabular}{|ll|c|c|c|}
\hline & \multicolumn{3}{|c|}{ \% SD39 } \\
\hline & \multicolumn{2}{|c|}{ Yes } & No & NS/REF \\
\hline a. & Feel stressed emotionally due to your caregiving & 76.0 & 24.0 & 0.0 \\
\hline responsibilities & & & \\
\hline b. & Feel stressed in trying to balance your job and family & 51.5 & 43.1 & 5.4 \\
\hline c. & Find it difficult to maintain a healthy diet & 28.9 & 67.4 & 3.7 \\
\hline d. & Find it difficult to get enough rest & 40.2 & 59.8 & 0.0 \\
\hline e. & Find it difficult to exercise regularly & 51.9 & 48.1 & 0.0 \\
\hline f. & Find it difficult to take care of your household \\
& responsibilities & 40.4 & 55.9 & 3.7 \\
\hline g. & Experience problems with your health & 19.0 & 81.0 & 0.0 \\
\hline h. & Find it difficult to make time to visit your doctor & 18.8 & 81.2 & 0.0 \\
\hline i. & Feel strained financially due to your caregiving \\
& responsibilities & 23.0 & 77.0 & 0.0 \\
\hline
\end{tabular}

Q12. How important is it to you to be able to provide care for your loved one so that they can keep living independently in their own home? Is it ...?

\begin{tabular}{|l|c|c|c|c|}
\hline & \% ALL & \% SD5 & \% SD26 & $\%$ SD39 \\
\hline Extremely important & 62.3 & 79.2 & 63.9 & 69.7 \\
\hline Very important & 29.5 & 20.8 & 30.6 & 23.0 \\
\hline Somewhat important & 2.6 & 0.0 & 2.7 & 0.0 \\
\hline Not very important & 2.1 & 0.0 & 2.7 & 3.7 \\
\hline Not at all important & 3.0 & 0.0 & 0.0 & 3.7 \\
\hline DK/REF & 0.4 & 0.0 & 0.0 & 0.0 \\
\hline
\end{tabular}


Q13. How likely do you think it is that you will provide any caregiving or assistance on an unpaid basis for an adult loved one in the future? Is it ...?

\begin{tabular}{|l|c|c|c|c|}
\hline & \% ALL & \% SD5 & \% SD26 & \% SD39 \\
\hline Extremely likely & 52.1 & 65.6 & 43.2 & 52.0 \\
\hline Very likely & 24.9 & 14.9 & 22.4 & 23.7 \\
\hline Somewhat likely & 10.0 & 9.8 & 18.2 & 11.5 \\
\hline Not very likely & 6.0 & 4.9 & 10.7 & 0.0 \\
\hline Not at all likely & 4.1 & 0.0 & 2.7 & 12.8 \\
\hline NS/REF & 3.0 & 4.7 & 2.7 & 0.0 \\
\hline
\end{tabular}

\section{SKIP TO QUESTION 26}

QUESTIONS 14-25 ARE FOR FORMER CAREGIVERS AND EMULATE THOSE FOR CURRENT CAREGIVERS. NOTE TO DATA PROGRAMMERS: DURING THE DATA PROCESSING QUESTIONS 2-25 NEED TO BE RUN AS IS AND ALSO COLLAPSED SO THAT THERE IS ONE SET OF DATA FOR EACH OF THESE QUESTIONS THAT AGGREGATES CURRENT AND FORMER CAREGIVERS TOGETHER.

Q14. Have you ever been a family caregiver providing UNPAID care to an adult loved one?

\begin{tabular}{|l|c|c|c|c|}
\hline & \% ALL & \% SD5 & \% SD26 & \% SD39 \\
\hline Yes & 45.9 & 54.0 & 50.0 & 40.1 \\
\hline No & 53.8 & 46.0 & 49.3 & 59.9 \\
\hline DK/REF & 0.3 & 0.0 & 0.8 & 0.0 \\
\hline
\end{tabular}

Q15. What is the age of the person you most recently provided care to?

\begin{tabular}{|l|c|c|c|c|}
\hline & \% ALL & \% SD5 & \% SD26 & \% SD39 \\
\hline Under 18 & 0.4 & 0.0 & 0.0 & 2.9 \\
\hline $18-49$ & 3.0 & 1.3 & 5.1 & 1.7 \\
\hline $50-59$ & 4.3 & 7.4 & 0.0 & 1.7 \\
\hline $60-64$ & 4.3 & 2.6 & 4.9 & 1.7 \\
\hline $65+$ & 84.9 & 85.9 & 86.7 & 86.8 \\
\hline NS/REF & 3.0 & 2.8 & 3.3 & 5.3 \\
\hline Mean Age & 77.7 & 76.9 & 78.8 & 79.1 \\
\hline
\end{tabular}


Q16. Thinking about the person you most recently provided care to, which of the following activities did you provide? Did you ...?

\begin{tabular}{|l|c|c|c|}
\hline & \multicolumn{3}{|c|}{$\%$ ALL } \\
\hline & Yes & No & NS/REF \\
\hline a. Assist with bathing or dressing & 52.4 & 47.6 & 0.0 \\
\hline b. Provide transportation to appointments & 85.3 & 14.7 & 0.0 \\
\hline c. Take them shopping or shopping for them & 87.2 & 12.6 & 0.2 \\
\hline d. Help them with meals & 80.8 & 19.0 & 0.2 \\
\hline e. Help them to manage finances & 63.5 & 36.5 & 0.0 \\
\hline f. Aid with household chores & 83.0 & 16.3 & 0.6 \\
\hline g. Provide social activities or companionship & 90.3 & 8.6 & 1.0 \\
\hline h. Oversee their medication management & 70.1 & 29.9 & 0.0 \\
\hline i. Have responsibility for other medical or nursing tasks & 62.3 & 36.0 & 1.6 \\
\hline
\end{tabular}

\begin{tabular}{|l|c|c|c|}
\hline & \multicolumn{3}{|c|}{ \% SD5 } \\
\hline & Yes & No & NS/REF \\
\hline a. Assisting with bathing or dressing & 45.4 & 54.6 & 0.0 \\
\hline b. Providing transportation to appointments & 90.0 & 10.0 & 0.0 \\
\hline c. Taking them shopping or shopping for them & 88.9 & 11.1 & 0.0 \\
\hline d. Helping them with meals & 81.4 & 18.6 & 0.0 \\
\hline e. Helping them to manage finances & 59.8 & 40.2 & 0.0 \\
\hline f. Aiding with household chores & 82.1 & 16.5 & 1.4 \\
\hline g. Providing social activities or companionship & 90.5 & 8.2 & 1.3 \\
\hline h. Overseeing their medication management & 68.1 & 31.9 & 0.0 \\
\hline i. Being responsible for other medical or nursing tasks & 58.4 & 40.2 & 1.4 \\
\hline
\end{tabular}

\begin{tabular}{|l|c|c|c|}
\hline & \multicolumn{3}{|c|}{ \% SD26 } \\
\hline & Yes & No & NS/REF \\
\hline a. Assisting with bathing or dressing & 53.8 & 46.2 & 0.0 \\
\hline b. Providing transportation to appointments & 88.4 & 11.6 & 0.0 \\
\hline c. Taking them shopping or shopping for them & 93.4 & 6.6 & 0.0 \\
\hline d. Helping them with meals & 79.3 & 19.0 & 1.7 \\
\hline e. Helping them to manage finances & 72.8 & 27.2 & 0.0 \\
\hline f. Aiding with household chores & 81.0 & 17.3 & 1.7 \\
\hline g. Providing social activities or companionship & 91.8 & 4.8 & 3.4 \\
\hline h. Overseeing their medication management & 80.3 & 19.7 & 0.0 \\
\hline i. $\quad$ Being responsible for other medical or nursing tasks & 60.6 & 36.1 & 3.3 \\
\hline
\end{tabular}




\begin{tabular}{|ll|c|c|c|}
\hline & \multicolumn{3}{|c|}{ \% SD39 } \\
\hline & Yes & No & NS/REF \\
\hline a. & Assisting with bathing or dressing & 54.8 & 45.2 & 0.0 \\
\hline b. $\quad$ Providing transportation to appointments & 79.8 & 20.2 & 0.0 \\
\hline c. & Taking them shopping or shopping for them & 87.6 & 12.4 & 0.0 \\
\hline d. Helping them with meals & 82.4 & 17.6 & 0.0 \\
\hline e. $\quad$ Helping them to manage finances & 69.9 & 30.1 & 0.0 \\
\hline f. $\quad$ Aiding with household chores & 78.3 & 19.9 & 1.9 \\
\hline g. & Providing social activities or companionship & 92.7 & 7.3 & 0.0 \\
\hline h. $\quad$ Overseeing their medication management & 66.6 & 33.4 & 0.0 \\
\hline i. $\quad$ Being responsible for other medical or nursing tasks & 44.7 & 53.6 & 1.7 \\
\hline
\end{tabular}

Q16b1. Did you drive your loved one to medical appointments?

\begin{tabular}{|l|c|c|c|c|}
\hline & \% ALL & \% SD5 & \% SD26 & \% SD39 \\
\hline Yes & 90.7 & 93.9 & 87.0 & 93.5 \\
\hline No & 9.3 & 6.1 & 13.0 & 6.5 \\
\hline DK/REF & 0.0 & 0.0 & 0.0 & 0.0 \\
\hline
\end{tabular}

Q16c. How often did you [a-i]:

\begin{tabular}{|ll|c|c|c|c|}
\hline & \multicolumn{4}{|c|}{$\%$ ALL } \\
\hline & Daily & Weekly & Monthly & NS/REF \\
\hline a. & Assist with bathing or dressing & 63.7 & 23.2 & 6.2 & 6.9 \\
\hline b. Provide transportation to appointments & 13.1 & 45.1 & 36.0 & 5.9 \\
\hline c. & Take them shopping or shop for them & 10.7 & 67.1 & 17.3 & 4.8 \\
\hline d. Help them with meals & 72.2 & 18.6 & 5.1 & 4.1 \\
\hline e. & Help them to manage finances & 35.9 & 28.5 & 28.5 & 7.1 \\
\hline f. Aid with household chores & 61.2 & 28.0 & 6.8 & 4.0 \\
\hline g. Provide social activities or companionship & 68.5 & 22.9 & 5.8 & 2.7 \\
\hline h. Oversee their medication management & 74.1 & 17.7 & 3.8 & 4.4 \\
\hline i. & Be responsible for other medical or nursing \\
& tasks & 58.1 & 22.8 & 11.4 & 7.8 \\
\hline
\end{tabular}

\begin{tabular}{|ll|c|c|c|c|}
\hline & \multicolumn{4}{|c|}{$\%$ SD5 } \\
\hline & Daily & Weekly & Monthly & NS/REF \\
\hline a. & Assist with bathing or dressing & 59.1 & 28.5 & 3.1 & 9.3 \\
\hline b. & Provide transportation to appointments & 18.4 & 47.9 & 25.6 & 8.1 \\
\hline c. & Take them shopping or shop for them & 12.5 & 60.6 & 20.7 & 6.2 \\
\hline d. & Help them with meals & 77.8 & 12.0 & 3.5 & 6.7 \\
\hline e. & Help them to manage finances & 37.4 & 20.4 & 23.8 & 18.3 \\
\hline f. & Aid with household chores & 55.5 & 27.2 & 10.1 & 7.2 \\
\hline g. Provide social activities or companionship & 67.3 & 26.6 & 3.0 & 3.1 \\
\hline h. Oversee their medication management & 72.3 & 15.9 & 5.8 & 6.0 \\
\hline i. & $\begin{array}{l}\text { Be responsible for other medical or nursing } \\
\text { tasks }\end{array}$ & 64.6 & 19.1 & 9.2 & 7.1 \\
\hline
\end{tabular}




\begin{tabular}{|ll|c|c|c|c|}
\hline & \multicolumn{4}{|c|}{ \% SD26 } \\
\hline a. & Assist with bathing or dressing & Daily & Weekly & Monthly & NS/REF \\
\hline b. & Provide transportation to appointments & 10.5 & 29.5 & 3.1 & 12.6 \\
\hline c. & Take them shopping or shop for them & 12.3 & 64.7 & 25.3 & 6.5 \\
\hline d. & Help them with meals & 68.6 & 20.9 & 4.1 & 5.5 \\
\hline e. & Help them to manage finances & 25.9 & 31.6 & 28.9 & 6.4 \\
\hline f. & Aid with household chores & 64.1 & 27.7 & 4.1 & 4.7 \\
\hline g. & Provide social activities or companionship & 58.6 & 30.1 & 7.6 & 3.7 \\
\hline h. & Oversee their medication management & 67.1 & 28.8 & 2.0 & 2.1 \\
\hline i. & Be responsible for other medical or nursing \\
& tasks & 46.4 & 34.7 & 8.0 & 10.8 \\
\hline
\end{tabular}

\begin{tabular}{|c|c|c|c|c|}
\hline & \multicolumn{4}{|c|}{$\%$ SD39 } \\
\hline & Daily & Weekly & Monthly & NS/REF \\
\hline a. Assist with bathing or dressing & 48.4 & 34.2 & 11.0 & 6.3 \\
\hline b. Provide transportation to appointments & 6.9 & 53.8 & 35.0 & 4.3 \\
\hline c. Take them shopping or shop for them & 7.6 & 72.5 & 19.9 & 0.0 \\
\hline d. Help them with meals & 64.2 & 25.6 & 10.2 & 0.0 \\
\hline e. Help them to manage finances & 28.0 & 37.6 & 34.5 & 0.0 \\
\hline f. $\quad$ Aid with household chores & 66.7 & 24.6 & 6.3 & 2.4 \\
\hline g. Provide social activities or companionship & 64.4 & 30.2 & 5.5 & 0.0 \\
\hline h. Oversee their medication management & 63.3 & 26.0 & 2.6 & 8.1 \\
\hline $\begin{array}{l}\text { i. Be responsible for other medical or nursing } \\
\text { tasks }\end{array}$ & 68.7 & 15.1 & 4.2 & 12.1 \\
\hline
\end{tabular}

Q17. Thinking about the person you provided care to, about how long would it have taken you, in minutes, to this person to:

\begin{tabular}{|l|c|c|c|}
\hline & \multicolumn{3}{|c|}{ \% ALL } \\
\hline & The nearest hospital & $\begin{array}{c}\text { A doctor's office for } \\
\text { primary care }\end{array}$ & $\begin{array}{c}\text { A doctor's office for } \\
\text { specialty care }\end{array}$ \\
\hline Less than 5 minutes & 3.1 & 1.7 & 1.2 \\
\hline 5-10 minutes & 20.5 & 12.7 & 7.2 \\
\hline 11-15 minutes & 20.0 & 20.3 & 13.8 \\
\hline 16-30 minutes & 36.6 & 38.3 & 34.7 \\
\hline 31-45 minutes & 9.7 & 10.1 & 14.7 \\
\hline 46 minutes to an hour & 3.6 & 7.3 & 9.5 \\
\hline More than 1 hour & 3.1 & 3.7 & 9.6 \\
\hline NS/REF & 3.4 & 5.9 & 9.3 \\
\hline
\end{tabular}




\begin{tabular}{|l|c|c|c|}
\hline & \multicolumn{3}{|c|}{ \% SD5 } \\
\hline The nearest hospital & $\begin{array}{c}\text { A doctor's office for } \\
\text { primary care }\end{array}$ & $\begin{array}{c}\text { A doctor's office for } \\
\text { specialty care }\end{array}$ \\
\hline Less than 5 minutes & 3.1 & 2.8 & 1.4 \\
\hline 5-10 minutes & 8.8 & 10.2 & 4.5 \\
\hline 11-15 minutes & 18.0 & 16.9 & 10.0 \\
\hline 16-30 minutes & 46.4 & 39.1 & 29.5 \\
\hline 31-45 minutes & 8.69 & 7.7 & 12.7 \\
\hline 46 minutes to an hour & 2.7 & 8.3 & 9.6 \\
\hline More than 1 hour & 8.3 & 8.2 & 18.3 \\
\hline NS/REF & 4.1 & 6.9 & 14.0 \\
\hline
\end{tabular}

\begin{tabular}{|l|c|c|c|}
\hline & \multicolumn{3}{|c|}{ \% SD26 } \\
\hline The nearest hospital & $\begin{array}{c}\text { A doctor's office for } \\
\text { primary care }\end{array}$ & $\begin{array}{c}\text { A doctor's office for } \\
\text { specialty care }\end{array}$ \\
\hline Less than 5 minutes & 4.9 & 4.9 & 3.3 \\
\hline 5-10 minutes & 18.9 & 12.3 & 7.4 \\
\hline 11-15 minutes & 14.6 & 11.6 & 6.7 \\
\hline 16-30 minutes & 41.6 & 40.6 & 34.1 \\
\hline 31-45 minutes & 8.3 & 6.6 & 16.1 \\
\hline 46 minutes to an hour & 5.0 & 11.6 & 13.3 \\
\hline More than 1 hour & 3.3 & 7.4 & 12.4 \\
\hline NS/REF & 3.4 & 5.0 & 6.8 \\
\hline
\end{tabular}

\begin{tabular}{|l|c|c|c|}
\hline & \multicolumn{3}{|c|}{ \% SD39 } \\
\hline The nearest hospital & $\begin{array}{c}\text { A doctor's office for } \\
\text { primary care }\end{array}$ & $\begin{array}{c}\text { A doctor's office for } \\
\text { specialty care }\end{array}$ \\
\hline Less than 5 minutes & 1.7 & 0.0 & 0.0 \\
\hline $5-10$ minutes & 24.5 & 12.1 & 8.7 \\
\hline $11-15$ minutes & 28.6 & 26.6 & 21.4 \\
\hline 16-30 minutes & 30.7 & 39.8 & 35.0 \\
\hline 31-45 minutes & 7.5 & 7.2 & 10.8 \\
\hline 46 minutes to an hour & 0.0 & 1.7 & 3.3 \\
\hline More than 1 hour & 3.4 & 3.3 & 6.9 \\
\hline NS/REF & 3.6 & 9.2 & 14.0 \\
\hline
\end{tabular}

Q18. Thinking about the person you provide care to, and the county they live in, do you need to travel out of their county to get to ...?

\begin{tabular}{|c|c|c|c|}
\hline & \multicolumn{3}{|c|}{$\%$ ALL } \\
\hline & Yes & No & NS/REF \\
\hline a. The nearest hospital & 22.3 & 76.5 & 1.2 \\
\hline b. A doctor's office for primary care & 21.2 & 77.2 & 1.6 \\
\hline $\begin{array}{l}\text { c. A doctor's office for specialty care, like a cardiologist, } \\
\text { neurologist, or other specialist }\end{array}$ & 40.5 & 56.3 & 3.3 \\
\hline
\end{tabular}




\begin{tabular}{|c|c|c|c|}
\hline & \multicolumn{3}{|c|}{$\%$ SD5 } \\
\hline & Yes & No & NS/REF \\
\hline a. The nearest hospital & 35.1 & 62.2 & 2.7 \\
\hline b. A doctor's office for primary care & 34.4 & 62.9 & 2.7 \\
\hline $\begin{array}{l}\text { c. A doctor's office for specialty care, like a cardiologist, } \\
\text { neurologist, or other specialist }\end{array}$ & 49.6 & 47.7 & 2.7 \\
\hline
\end{tabular}

\begin{tabular}{|ll|c|c|c|}
\hline & \multicolumn{3}{|c|}{ \% SD26 } \\
\hline & Yes & No & NS/REF \\
\hline a. & The nearest hospital & 17.3 & 82.7 & 0.0 \\
\hline b. A doctor's office for primary care & 17.4 & 82.6 & 0.0 \\
\hline $\begin{array}{l}\text { c. A doctor's office for specialty care, like a cardiologist, } \\
\text { neurologist, or other specialist }\end{array}$ & 40.2 & 53.2 & 6.7 \\
\hline
\end{tabular}

\begin{tabular}{|l|c|c|c|}
\hline & \multicolumn{3}{|c|}{ \% SD39 } \\
\hline & Yes & No & NS/REF \\
\hline a. The nearest hospital & 6.9 & 91.4 & 1.7 \\
\hline b. A doctor's office for primary care & 15.6 & 79.2 & 5.3 \\
\hline $\begin{array}{l}\text { c. A doctor's office for specialty care, like a cardiologist, } \\
\text { neurologist, or other specialist }\end{array}$ & 22.2 & 72.5 & 5.3 \\
\hline
\end{tabular}

Q19. Have you ever been employed either full- or part-time while providing care to your adult loved one?

\begin{tabular}{|l|c|c|c|c|}
\hline & \% ALL & \% SD5 & \% SD26 & \% SD39 \\
\hline Yes & 58.3 & 55.3 & 59.8 & 55.6 \\
\hline No & 41.7 & 44.7 & 40.2 & 44.4 \\
\hline DK/REF & 0.0 & 0.0 & 0.0 & 0.0 \\
\hline
\end{tabular}

Q20. Now thinking again about the adult loved one you most recently provided care to, did you ever have to ...?

\begin{tabular}{|l|c|c|c|}
\hline & \multicolumn{3}{|c|}{$\%$ ALL } \\
\hline $\begin{array}{l}\text { a. Go into work early or late, or take time off to provide care } \\
\text { for a loved one }\end{array}$ & 71.8 & 28.2 & 0.0 \\
\hline $\begin{array}{l}\text { b. Take a leave of absence from your job to provide care for } \\
\text { a loved one }\end{array}$ & 28.6 & 71.4 & 0.0 \\
\hline $\begin{array}{l}\text { c. Go from working full-time to part-time to provide care for } \\
\text { a loved one }\end{array}$ & 18.2 & 81.5 & 0.4 \\
\hline d. Give up working entirely to provide care for a loved one & 15.3 & 84.0 & 0.7 \\
\hline
\end{tabular}




\begin{tabular}{|l|c|c|c|}
\hline & \multicolumn{3}{|c|}{$\%$ SD5 } \\
\hline $\begin{array}{l}\text { a. Go into work early or late, or take time off to provide care } \\
\text { for a loved one }\end{array}$ & 65.6 & 34.4 & 0.0 \\
\hline $\begin{array}{l}\text { b. Take a leave of absence from your job to provide care for } \\
\text { a loved one }\end{array}$ & 41.8 & 58.2 & 0.0 \\
\hline $\begin{array}{l}\text { c. Go from working full-time to part-time to provide care for } \\
\text { a loved one }\end{array}$ & 24.6 & 72.9 & 2.5 \\
\hline d. Give up working entirely to provide care for a loved one & 18.8 & 81.2 & 0.0 \\
\hline
\end{tabular}

\begin{tabular}{|l|c|c|c|}
\hline & \multicolumn{3}{|c|}{$\%$ SD26 } \\
\hline $\begin{array}{l}\text { a. Go into work early or late, or take time off to provide care } \\
\text { for a loved one }\end{array}$ & 66.6 & 33.4 & 0.0 \\
\hline $\begin{array}{l}\text { b. Take a leave of absence from your job to provide care for } \\
\text { a loved one }\end{array}$ & 16.6 & 83.4 & 0.0 \\
\hline $\begin{array}{l}\text { c. Go from working full-time to part-time to provide care for } \\
\text { a loved one }\end{array}$ & 11.0 & 89.0 & 0.0 \\
\hline d. Give up working entirely to provide care for a loved one & 20.7 & 73.8 & 5.5 \\
\hline
\end{tabular}

\begin{tabular}{|l|c|c|c|}
\hline & \multicolumn{3}{|c|}{$\%$ SD39 } \\
\hline $\begin{array}{l}\text { a. Go into work early or late, or take time off to provide care } \\
\text { for a loved one }\end{array}$ & 77.9 & 22.1 & 0.0 \\
\hline $\begin{array}{l}\text { b. Take a leave of absence from your job to provide care for } \\
\text { a loved one }\end{array}$ & 21.0 & 79.0 & 0.0 \\
\hline $\begin{array}{l}\text { c. Go from working full-time to part-time to provide care for } \\
\text { a loved one }\end{array}$ & 21.9 & 78.1 & 0.0 \\
\hline d. Give up working entirely to provide care for a loved one & 15.5 & 84.5 & 0.0 \\
\hline
\end{tabular}

Q21. [IF Q20A=YES ASK] You mentioned that you had to go into work early or late, or take time off to provide care to an adult loved one. How often would you say you had to take time away from work or adjust your work schedule to provide care for someone? Would you say ...?

\begin{tabular}{|l|c|c|c|c|}
\hline & \% ALL & \% SD5 & \% SD26 & $\%$ SD39 \\
\hline More than once per week & 13.1 & 12.7 & 0.0 & 19.0 \\
\hline Once per week & 10.9 & 16.5 & 4.3 & 8.0 \\
\hline $\begin{array}{l}\text { Several times per month but less often } \\
\text { than once per week }\end{array}$ & 25.7 & 19.4 & 31.7 & 24.4 \\
\hline Once per month & 24.1 & 20.1 & 14.3 & 32.8 \\
\hline Less than once per month & 19.2 & 15.9 & 41.2 & 11.9 \\
\hline DK/REF & 7.0 & 15.3 & 8.5 & 3.9 \\
\hline
\end{tabular}


Q22. Now thinking again about the adult loved one you have most recently provided care to, did you ever have to ...?

\begin{tabular}{|c|c|c|c|}
\hline & \multicolumn{3}{|c|}{$\%$ ALL } \\
\hline & Yes & No & NS/REF \\
\hline a. Use your own money to help provide care & 59.3 & 38.5 & 2.3 \\
\hline b. Make changes to your home for the loved one in your care & 34.3 & 65.5 & 0.2 \\
\hline $\begin{array}{l}\text { c. Move into another home to accommodate the loved one in } \\
\text { your care }\end{array}$ & 14.6 & 85.0 & 0.4 \\
\hline
\end{tabular}

\begin{tabular}{|l|c|c|c|}
\hline & \multicolumn{3}{|c|}{$\%$ SD5 } \\
\hline & Yes & No & NS/REF \\
\hline a. Use your own money to help provide care & 47.8 & 46.7 & 5.5 \\
\hline b. Make changes to your home for the loved one in your care & 40.8 & 59.2 & 0.0 \\
\hline $\begin{array}{l}\text { c. Move into another home to accommodate the loved one in } \\
\text { your care }\end{array}$ & 19.1 & 80.9 & 0.0 \\
\hline
\end{tabular}

\begin{tabular}{|l|c|c|c|}
\hline & \multicolumn{3}{|c|}{ \% SD26 } \\
\hline \multicolumn{1}{|c|}{ Use your own money to help provide care } & Yes & No & NS/REF \\
\hline a. & 64.5 & 33.9 & 1.6 \\
\hline $\begin{array}{l}\text { c. } \text { Make changes to your home for the loved one in your care } \\
\text { your care }\end{array}$ & 33.3 & 66.7 & 0.0 \\
\hline
\end{tabular}

\begin{tabular}{|l|c|c|c|}
\hline & \multicolumn{3}{|c|}{ \% SD39 } \\
\hline & Yes & No & NS/REF \\
\hline a. Use your own money to help provide care & 54.5 & 38.5 & 2.3 \\
\hline b. Make changes to your home for the loved one in your care & 24.1 & 75.9 & 0.0 \\
\hline \begin{tabular}{l} 
c. $\begin{array}{l}\text { Move into another home to accommodate the loved one in } \\
\text { your care }\end{array}$ \\
\hline
\end{tabular} & 25.7 & 74.3 & 0.0 \\
\hline
\end{tabular}


Q23. As a caregiver did you ever ...?

\begin{tabular}{|c|c|c|c|}
\hline & \multicolumn{3}{|c|}{$\%$ ALL } \\
\hline & Yes & No & NS/REF \\
\hline $\begin{array}{l}\text { a. Feel stressed emotionally due to your caregiving } \\
\text { responsibilities }\end{array}$ & 67.6 & 32.0 & 0.4 \\
\hline b. Feel stressed in trying to balance your job and family & 67.2 & 32.8 & 0.0 \\
\hline c. Find it difficult to maintain a healthy diet & 31.8 & 67.4 & 0.8 \\
\hline d. Find it difficult to get enough rest & 58.5 & 41.3 & 0.2 \\
\hline e. Find it difficult to exercise regularly & 47.6 & 51.4 & 1.0 \\
\hline $\begin{array}{l}\text { f. Find it difficult to take care of your household } \\
\text { responsibilities }\end{array}$ & 45.3 & 54.0 & 0.6 \\
\hline g. Experience problems with your health & 30.0 & 69.8 & 0.2 \\
\hline h. Find it difficult to make time to visit your doctor & 19.3 & 80.3 & 0.4 \\
\hline $\begin{array}{l}\text { i. Feel strained financially due to your caregiving } \\
\text { responsibilities }\end{array}$ & 25.7 & 73.9 & 0.4 \\
\hline j. Feel stressed about anything else & 31.2 & 67.7 & 1.0 \\
\hline
\end{tabular}

\begin{tabular}{|c|c|c|c|}
\hline & \multicolumn{3}{|c|}{$\% \mathrm{SD} 5$} \\
\hline & Yes & No & NS/REF \\
\hline $\begin{array}{l}\text { a. Feel stressed emotionally due to your caregiving } \\
\text { responsibilities }\end{array}$ & 70.3 & 28.3 & 1.4 \\
\hline b. Feel stressed in trying to balance your job and family & 70.5 & 29.5 & 0.0 \\
\hline c. Find it difficult to maintain a healthy diet & 36.5 & 63.5 & 0.0 \\
\hline d. Find it difficult to get enough rest & 55.2 & 44.8 & 0.0 \\
\hline e. Find it difficult to exercise regularly & 57.1 & 42.9 & 0.0 \\
\hline $\begin{array}{l}\text { f. Find it difficult to take care of your household } \\
\text { responsibilities }\end{array}$ & 46.5 & 52.2 & 1.3 \\
\hline g. Experience problems with your health & 36.7 & 63.3 & 0.0 \\
\hline h. Find it difficult to make time to visit your doctor & 24.3 & 75.7 & 0.0 \\
\hline $\begin{array}{l}\text { i. Feel strained financially due to your caregiving } \\
\text { responsibilities }\end{array}$ & 29.7 & 70.3 & 0.0 \\
\hline j. Feel stressed about anything else & 30.8 & 67.8 & 1.4 \\
\hline
\end{tabular}




\begin{tabular}{|c|c|c|c|}
\hline & \multicolumn{3}{|c|}{$\%$ SD26 } \\
\hline & Yes & No & NS/REF \\
\hline $\begin{array}{l}\text { a. Feel stressed emotionally due to your caregiving } \\
\text { responsibilities }\end{array}$ & 60.2 & 39.8 & 0.0 \\
\hline b. Feel stressed in trying to balance your job and family & 49.1 & 50.9 & 0.0 \\
\hline c. Find it difficult to maintain a healthy diet & 29.3 & 69.0 & 1.7 \\
\hline d. Find it difficult to get enough rest & 56.6 & 41.7 & 1.7 \\
\hline e. Find it difficult to exercise regularly & 36.0 & 62.3 & 1.7 \\
\hline $\begin{array}{l}\text { f. Find it difficult to take care of your household } \\
\text { responsibilities }\end{array}$ & 38.6 & 61.4 & 0.0 \\
\hline g. Experience problems with your health & 34.4 & 65.6 & 0.0 \\
\hline h. Find it difficult to make time to visit your doctor & 12.9 & 85.4 & 1.7 \\
\hline $\begin{array}{l}\text { i. Feel strained financially due to your caregiving } \\
\text { responsibilities }\end{array}$ & 27.9 & 70.5 & 1.7 \\
\hline j. Feel stressed about anything else & 17.1 & 79.5 & 3.4 \\
\hline
\end{tabular}

\begin{tabular}{|ll|c|c|c|}
\hline & \multicolumn{3}{|c|}{$\%$ SD39 } \\
\hline & Yes & No & NS/REF \\
\hline a. & Feel stressed emotionally due to your caregiving & 73.1 & 26.9 & 0.0 \\
\hline responsibilities & & 74.3 & 25.7 & 0.0 \\
\hline b. Feel stressed in trying to balance your job and family & 20.8 & 77.3 & 1.9 \\
\hline c. & Find it difficult to maintain a healthy diet & 62.6 & 37.4 & 0.0 \\
\hline d. Find it difficult to get enough rest & 47.3 & 51.0 & 1.7 \\
\hline e. & Find it difficult to exercise regularly & 40.8 & 59.2 & 0.0 \\
\hline f. $\quad$ Find it difficult to take care of your household & & & 0.0 \\
\hline responsibilities & Experience problems with your health & 19.3 & 80.7 & 0.0 \\
\hline h. & Find it difficult to make time to visit your doctor & 16.0 & 84.0 & 0.0 \\
\hline i. $\quad$ Feel strained financially due to your caregiving & 15.3 & 84.7 & 0.0 \\
\hline responsibilities & 332.6 & 65.5 & 1.9 \\
\hline j. & Feel stressed about anything else & & & \\
\hline
\end{tabular}

Q24. How important was it to you to be able to provide care for your loved one so that they can keep living independently in their own home? Is it ...?

\begin{tabular}{|l|c|c|c|c|}
\hline & \% ALL & \% SD5 & \% SD26 & \% SD39 \\
\hline Extremely important & 54.9 & 57.3 & 65.2 & 43.3 \\
\hline Very important & 34.6 & 34.2 & 23.1 & 36.3 \\
\hline Somewhat important & 6.2 & 4.4 & 8.2 & 15.0 \\
\hline Not very important & 0.8 & 0.0 & 0.0 & 0.0 \\
\hline Not at all important & 1.6 & 2.7 & 1.7 & 1.9 \\
\hline DK/REF & 1.8 & 1.3 & 1.7 & 3.5 \\
\hline
\end{tabular}


Q25. How likely do you think it is that you will provide any caregiving or assistance on an unpaid basis for an adult loved one in the future? Is it ...?

\begin{tabular}{|l|c|c|c|c|}
\hline & \% ALL & \% SD5 & \% SD26 & \% SD39 \\
\hline Extremely likely & 18.7 & 11.9 & 16.2 & 11.5 \\
\hline Very likely & 21.7 & 25.1 & 13.9 & 14.3 \\
\hline Somewhat likely & 19.9 & 15.5 & 26.1 & 28.2 \\
\hline Not very likely & 16.2 & 17.7 & 17.9 & 17.4 \\
\hline Not at all likely & 21.3 & 26.8 & 17.5 & 26.6 \\
\hline NS/REF & 2.3 & 3.1 & 8.4 & 1.9 \\
\hline
\end{tabular}

\section{CURRENT/FORMER CAREGIVERS}

Q1_Q14. Are you currently/have you ever been a family caregiver providing UNPAID care to an adult loved one?

\begin{tabular}{|l|c|c|c|c|}
\hline & \% ALL & \% SD5 & \% SD26 & \% SD39 \\
\hline Yes & 55.6 & 60.0 & 61.8 & 48.8 \\
\hline No & 44.4 & 40.0 & 38.2 & 51.2 \\
\hline DK/REF & 0.0 & 0.0 & 0.0 & 0.0 \\
\hline
\end{tabular}

Q2_Q15. What is/was the age of the person you are providing care for/who you most recently provided care to?

\begin{tabular}{|l|c|c|c|c|}
\hline & \% ALL & \% SD5 & \% SD26 & \% SD39 \\
\hline Under 18 & 0.3 & 0.0 & 0.0 & 2.0 \\
\hline $18-49$ & 4.4 & 3.1 & 5.2 & 8.5 \\
\hline $50-59$ & 4.4 & 5.8 & 2.0 & 1.2 \\
\hline $60-64$ & 5.3 & 5.2 & 4.0 & 2.3 \\
\hline $65+$ & 83.4 & 83.8 & 85.2 & 82.3 \\
\hline NS/REF & 2.2 & 2.2 & 3.5 & 3.7 \\
\hline Mean Age & 77.1 & 76.6 & 77.9 & 77.5 \\
\hline
\end{tabular}

Q3_Q16. Thinking about the person you are providing care to the most/you most recently provided care to, which of the following activities do/did you provide? Do/Did you ...?

\begin{tabular}{|l|c|c|c|}
\hline & \multicolumn{3}{|c|}{$\%$ ALL } \\
\hline & Yes & No & NS/REF \\
\hline a. Assist with bathing or dressing & 48.8 & 50.8 & 0.4 \\
\hline b. Provide transportation to appointments & 83.9 & 16.1 & 0.0 \\
\hline c. Take them shopping or shopping for them & 87.1 & 12.8 & 0.1 \\
\hline d. Help them with meals & 79.2 & 20.5 & 0.2 \\
\hline e. Help them to manage finances & 66.7 & 32.9 & 0.4 \\
\hline f. Aid with household chores & 82.9 & 16.7 & 0.4 \\
\hline g. Provide social activities/companionship & 90.1 & 9.1 & 0.8 \\
\hline j. Oversee their medication management & 69.4 & 30.4 & 0.1 \\
\hline k. Take responsibility for other medical or nursing tasks & 64.3 & 34.1 & 1.6 \\
\hline
\end{tabular}




\begin{tabular}{|l|c|c|c|}
\hline & \multicolumn{3}{|c|}{$\%$ SD5 } \\
\hline & Yes & No & NS/REF \\
\hline a. Assist with bathing or dressing & 48.6 & 50.3 & 1.1 \\
\hline b. Provide transportation to appointments & 90.0 & 10.0 & 0.0 \\
\hline c. Take them shopping or shopping for them & 91.3 & 8.7 & 0.0 \\
\hline d. Help them with meals & 79.9 & 20.1 & 0.0 \\
\hline e. Help them to manage finances & 67.5 & 32.5 & 0.0 \\
\hline f. Aid with household chores & 82.8 & 16.1 & 1.1 \\
\hline g. Provide social activities/companionship & 92.6 & 6.4 & 1.0 \\
\hline j. Oversee their medication management & 68.6 & 31.4 & 0.0 \\
\hline k. Take responsibility for other medical or nursing tasks & 62.0 & 36.9 & 1.1 \\
\hline
\end{tabular}

\begin{tabular}{|l|c|c|c|}
\hline & \multicolumn{3}{|c|}{ \% SD26 } \\
\hline & Yes & No & NS/REF \\
\hline a. Assist with bathing or dressing & 49.4 & 49.6 & 1.0 \\
\hline b. Provide transportation to appointments & 91.3 & 8.7 & 0.0 \\
\hline c. Take them shopping or shopping for them & 91.3 & 8.7 & 0.0 \\
\hline d. Help them with meals & 76.8 & 22.2 & 1.0 \\
\hline e. Help them to manage finances & 72.7 & 26.3 & 1.0 \\
\hline f. Aid with household chores & 84.2 & 14.7 & 1.0 \\
\hline g. Provide social activities/companionship & 89.3 & 8.6 & 2.1 \\
\hline j. Oversee their medication management & 79.2 & 20.8 & 0.0 \\
\hline k. Take responsibility for other medical or nursing tasks & 63.5 & 33.5 & 3.1 \\
\hline
\end{tabular}

\begin{tabular}{|c|c|c|c|}
\hline & \multicolumn{3}{|c|}{$\%$ SD39 } \\
\hline & Yes & No & NS/REF \\
\hline a. Assist with bathing or dressing & 52.7 & 46.2 & 1.1 \\
\hline b. Provide transportation to appointments & 81.1 & 18.9 & 0.0 \\
\hline c. Take them shopping or shopping for them & 87.5 & 12.5 & 0.0 \\
\hline d. Help them with meals & 83.2 & 16.8 & 0.0 \\
\hline e. Help them to manage finances & 74.2 & 25.8 & 0.0 \\
\hline f. Aid with household chores & 80.3 & 18.4 & 1.3 \\
\hline g. Provide social activities/companionship & 89.8 & 10.2 & 0.0 \\
\hline j. Oversee their medication management & 66.1 & 33.9 & 0.0 \\
\hline k. Take responsibility for other medical or nursing tasks & 56.8 & 42.0 & 1.2 \\
\hline
\end{tabular}

Q3b1_Q16b1. Do/did you drive your loved one to medical appointments?

\begin{tabular}{|l|c|c|c|c|}
\hline & \% ALL & \% SD5 & \% SD26 & \% SD39 \\
\hline Yes & 92.7 & 94.1 & 91.2 & 95.6 \\
\hline No & 7.3 & 5.9 & 8.8 & 4.4 \\
\hline DK/REF & 0.0 & 0.0 & 0.0 & 0.0 \\
\hline
\end{tabular}


Q4_Q16C. How often did you [a-i]:

\begin{tabular}{|c|c|c|c|c|}
\hline & \multicolumn{4}{|c|}{$\%$ ALL } \\
\hline & Daily & Weekly & Monthly & NS/REF \\
\hline a. Assist with bathing or dressing & 61.2 & 24.4 & 8.3 & 6.2 \\
\hline b. Provide transportation to appointments & 13.0 & 43.0 & 38.0 & 6.1 \\
\hline c. Take them shopping or shop for them & 11.0 & 65.7 & 18.8 & 4.6 \\
\hline d. Help them with meals & 69.4 & 19.9 & 6.5 & 4.2 \\
\hline e. Help them to manage finances & 39.3 & 27.3 & 28.7 & 4.8 \\
\hline f. Aid with household chores & 58.6 & 29.6 & 8.2 & 3.5 \\
\hline g. Provide social activities or companionship & 66.9 & 24.7 & 5.9 & 2.5 \\
\hline h. Oversee their medication management & 70.4 & 18.5 & 6.8 & 4.4 \\
\hline $\begin{array}{l}\text { i. Be responsible for other medical or nursing } \\
\text { tasks }\end{array}$ & 52.7 & 25.0 & 14.7 & 7.6 \\
\hline
\end{tabular}

\begin{tabular}{|c|c|c|c|c|}
\hline & \multicolumn{4}{|c|}{$\%$ SD5 } \\
\hline & Daily & Weekly & Monthly & NS/REF \\
\hline a. Assist with bathing or dressing & 65.8 & 23.0 & 2.3 & 8.9 \\
\hline b. Provide transportation to appointments & 18.1 & 45.0 & 29.3 & 7.6 \\
\hline c. Take them shopping or shop for them & 13.0 & 58.3 & 24.0 & 4.7 \\
\hline d. Help them with meals & 77.1 & 10.8 & 4.1 & 8.0 \\
\hline e. Help them to manage finances & 40.7 & 22.1 & 24.5 & 12.7 \\
\hline f. Aid with household chores & 58.8 & 27.8 & 7.8 & 5.6 \\
\hline g. Provide social activities or companionship & 68.5 & 22.5 & 6.5 & 2.6 \\
\hline h. Oversee their medication management & 75.2 & 15.5 & 4.5 & 4.7 \\
\hline $\begin{array}{l}\text { i. Be responsible for other medical or nursing } \\
\text { tasks }\end{array}$ & 61.8 & 26.3 & 6.7 & 5.2 \\
\hline
\end{tabular}

\begin{tabular}{|l|c|c|c|c|}
\hline & \multicolumn{4}{|c|}{ \% SD26 } \\
\hline & Daily & Weekly & Monthly & NS/REF \\
\hline a. Assist with bathing or dressing & 55.7 & 29.6 & 6.2 & 8.5 \\
\hline b. Provide transportation to appointments & 13.4 & 46.7 & 32.5 & 7.4 \\
\hline c. $\quad$ Take them shopping or shop for them & 14.5 & 60.7 & 18.0 & 6.9 \\
\hline d. Help them with meals & 66.0 & 24.7 & 5.2 & 4.1 \\
\hline e. Help them to manage finances & 41.0 & 27.1 & 23.4 & 8.5 \\
\hline f. Aid with household chores & 56.3 & 31.6 & 9.6 & 2.5 \\
\hline g. Provide social activities or companionship & 57.7 & 31.7 & 7.1 & 3.5 \\
\hline h. Oversee their medication management & 61.5 & 29.6 & 7.6 & 1.3 \\
\hline $\begin{array}{l}\text { i. } \\
\text { Be responsible for other medical or nursing } \\
\text { tasks }\end{array}$ & 43.7 & 36.5 & 10.4 & 9.5 \\
\hline
\end{tabular}




\begin{tabular}{|ll|c|c|c|c|}
\hline & \multicolumn{4}{|c|}{ \% SD39 } \\
\hline & Daily & Weekly & Monthly & NS/REF \\
\hline a. & Assist with bathing or dressing & 46.8 & 33.6 & 15.1 & 4.5 \\
\hline b. & Provide transportation to appointments & 6.1 & 49.6 & 37.1 & 7.3 \\
\hline c. & Take them shopping or shop for them & 8.3 & 66.9 & 22.2 & 2.5 \\
\hline d. & Help them with meals & 60.3 & 25.4 & 11.6 & 2.8 \\
\hline e. & Help them to manage finances & 37.9 & 30.9 & 31.3 & 0.0 \\
\hline f. & Aid with household chores & 61.8 & 22.9 & 13.7 & 1.6 \\
\hline g. & Provide social activities or companionship & 65.1 & 28.0 & 6.8 & 0.0 \\
\hline h. & Oversee their medication management & 66.7 & 24.0 & 3.6 & 5.7 \\
\hline i. & Be responsible for other medical or nursing \\
& tasks & 54.5 & 23.2 & 15.7 & 6.6 \\
\hline
\end{tabular}

Q5_Q17. Thinking about the person you provide care to, about how long would it take you, in minutes, to this person to:

\begin{tabular}{|l|c|c|c|}
\hline & \multicolumn{3}{|c|}{ \% ALL } \\
\hline & The nearest hospital & $\begin{array}{c}\text { A doctor's office for } \\
\text { primary care }\end{array}$ & $\begin{array}{c}\text { A doctor's office for } \\
\text { specialty care }\end{array}$ \\
\hline Less than 5 minutes & 3.5 & 2.4 & 1.4 \\
\hline 5-10 minutes & 20.8 & 14.8 & 8.3 \\
\hline 11-15 minutes & 21.0 & 18.6 & 14.1 \\
\hline 16-30 minutes & 35.7 & 39.0 & 34.8 \\
\hline 31-45 minutes & 10.0 & 8.9 & 13.7 \\
\hline 46 minutes to 1 hour & 3.2 & 6.4 & 10.0 \\
\hline More than 1 hour & 3.2 & 4.9 & 10.3 \\
\hline DK/REF & 2.7 & 5.0 & 7.3 \\
\hline
\end{tabular}

\begin{tabular}{|l|c|c|c|}
\hline & \multicolumn{3}{|c|}{ \% SD5 } \\
\hline & The nearest hospital & $\begin{array}{c}\text { A doctor's office for } \\
\text { primary care }\end{array}$ & $\begin{array}{c}\text { A doctor's office for } \\
\text { specialty care }\end{array}$ \\
\hline Less than 5 minutes & 2.4 & 2.2 & 1.1 \\
\hline 5-10 minutes & 9.1 & 11.2 & 5.7 \\
\hline 11-15 minutes & 15.3 & 14.3 & 7.8 \\
\hline 16-30 minutes & 49.3 & 39.5 & 29.4 \\
\hline 31-45 minutes & 9.9 & 7.0 & 15.9 \\
\hline 46 minutes to an hour & 3.2 & 10.8 & 11.8 \\
\hline More than 1 hour & 3.2 & 8.6 & 17.5 \\
\hline NS/REF & 2.7 & 6.5 & 10.9 \\
\hline
\end{tabular}




\begin{tabular}{|l|c|c|c|}
\hline & \multicolumn{3}{|c|}{ \% SD26 } \\
\hline The nearest hospital & $\begin{array}{c}\text { A doctor's office for } \\
\text { primary care }\end{array}$ & $\begin{array}{c}\text { A doctor's office for } \\
\text { specialty care }\end{array}$ \\
\hline 5-10 minutes & 5.89 & 6.8 & 2.0 \\
\hline 11-15 minutes & 22.1 & 11.7 & 8.2 \\
\hline 16-30 minutes & 15.1 & 10.2 & 6.1 \\
\hline 31-45 minutes & 40.5 & 46.2 & 38.1 \\
\hline 46 minutes to an hour & 9.3 & 7.2 & 15.5 \\
\hline More than 1 hour & 3.1 & 7.2 & 14.1 \\
\hline NS/REF & 2.0 & 6.6 & 10.7 \\
\hline
\end{tabular}

\begin{tabular}{|l|c|c|c|}
\hline & \multicolumn{3}{|c|}{ \% SD39 } \\
\hline The nearest hospital & $\begin{array}{c}\text { A doctor's office for } \\
\text { primary care }\end{array}$ & $\begin{array}{c}\text { A doctor's office for } \\
\text { specialty care }\end{array}$ \\
\hline Less than 5 minutes & 1.2 & 0.0 & 1.1 \\
\hline 5-10 minutes & 31.7 & 16.6 & 8.3 \\
\hline 16-30 minutes & 28.4 & 23.5 & 20.9 \\
\hline 31-45 minutes & 24.9 & 40.0 & 34.3 \\
\hline 46 minutes to an hour & 6.7 & 5.0 & 7.5 \\
\hline More than 1 hour & 0.0 & 1.2 & 4.7 \\
\hline NS/REF & 2.4 & 6.1 & 8.7 \\
\hline
\end{tabular}

Q6_Q18. Thinking about the person you provide care to, and the county they live in, do you need to travel out of their county to get to ...?

\begin{tabular}{|c|c|c|c|}
\hline & \multicolumn{3}{|c|}{$\%$ ALL } \\
\hline & Yes & No & NS/REF \\
\hline a. The nearest hospital & 22.4 & 76.8 & 0.8 \\
\hline b. A doctor's office for primary care & 21.3 & 77.3 & 1.4 \\
\hline $\begin{array}{l}\text { c. A doctor's office for specialty care, like a cardiologist, } \\
\text { neurologist, or other specialist }\end{array}$ & 40.1 & 57.5 & 2.4 \\
\hline
\end{tabular}

\begin{tabular}{|l|c|c|c|}
\hline & \multicolumn{3}{|c|}{$\%$ SD5 } \\
\hline & Yes & No & NS/REF \\
\hline a. The nearest hospital & 39.6 & 58.3 & 2.1 \\
\hline b. A doctor's office for primary care & 36.9 & 59.9 & 3.2 \\
\hline $\begin{array}{l}\text { c. A doctor's office for specialty care, like a cardiologist, } \\
\text { neurologist, or other specialist }\end{array}$ & 54.3 & 43.6 & 2.1 \\
\hline
\end{tabular}

\begin{tabular}{|l|c|c|c|}
\hline & \multicolumn{3}{|c|}{$\%$ SD26 } \\
\hline & Yes & No & NS/REF \\
\hline a. The nearest hospital & 13.7 & 86.3 & 0.0 \\
\hline b. A doctor's office for primary care & 14.8 & 84.2 & 1.0 \\
\hline $\begin{array}{l}\text { c. A doctor's office for specialty care, like a cardiologist, } \\
\text { neurologist, or other specialist }\end{array}$ & 40.9 & 55.0 & 4.1 \\
\hline
\end{tabular}




\begin{tabular}{|c|c|c|c|}
\hline & \multicolumn{3}{|c|}{$\%$ SD39 } \\
\hline & Yes & No & NS/REF \\
\hline a. The nearest hospital & 7.2 & 91.7 & 1.2 \\
\hline b. A doctor's office for primary care & 15.9 & 80.4 & 3.7 \\
\hline $\begin{array}{l}\text { c. A doctor's office for specialty care, like a cardiologist, } \\
\text { neurologist, or other specialist }\end{array}$ & 22.9 & 73.5 & 3.7 \\
\hline
\end{tabular}

Q7_Q19. Have you ever been employed either full- or part-time while providing care to your adult loved one?

\begin{tabular}{|l|c|c|c|c|}
\hline & \% ALL & \% SD5 & \% SD26 & \% SD39 \\
\hline Yes & 59.2 & 57.7 & 57.8 & 59.3 \\
\hline No & 40.8 & 42.3 & 42.2 & 40.7 \\
\hline DK/REF & 0.0 & 0.0 & 0.0 & 0.0 \\
\hline
\end{tabular}

Q8_Q20. Now thinking again about the adult loved one you are currently providing/most recently provided care to, did you ever have to ...?

\begin{tabular}{|l|c|c|c|}
\hline & \multicolumn{3}{|c|}{ \% ALL } \\
\hline $\begin{array}{l}\text { a. Go into work early or late, or take time off to provide care } \\
\text { for a loved one }\end{array}$ & 73.3 & 26.7 & 0.0 \\
\hline $\begin{array}{l}\text { b. Take a leave of absence from your job to provide care for } \\
\text { a loved one }\end{array}$ & 30.2 & 69.4 & 0.4 \\
\hline $\begin{array}{l}\text { c. Go from working full-time to part-time to provide care for } \\
\text { a loved one }\end{array}$ & 18.1 & 81.4 & 0.5 \\
\hline d. Give up working entirely to provide care for a loved one & 17.2 & 82.3 & 0.5 \\
\hline
\end{tabular}

\begin{tabular}{|l|c|c|c|}
\hline & \multicolumn{3}{|c|}{$\%$ SD5 } \\
\hline $\begin{array}{l}\text { a. Go into work early or late, or take time off to provide care } \\
\text { for a loved one }\end{array}$ & 70.6 & 29.4 & 0.0 \\
\hline $\begin{array}{l}\text { b. Take a leave of absence from your job to provide care for } \\
\text { a loved one }\end{array}$ & 49.0 & 51.0 & 0.0 \\
\hline $\begin{array}{l}\text { c. Go from working full-time to part-time to provide care for } \\
\text { a loved one }\end{array}$ & 24.6 & 73.5 & 1.9 \\
\hline d. Give up working entirely to provide care for a loved one & 22.1 & 77.9 & 0.0 \\
\hline
\end{tabular}

\begin{tabular}{|l|c|c|c|}
\hline & \multicolumn{3}{|c|}{ \% SD26 } \\
\hline $\begin{array}{l}\text { a. Go into work early or late, or take time off to provide care } \\
\text { for a loved one }\end{array}$ & 66.3 & 33.7 & 0.0 \\
\hline $\begin{array}{l}\text { b. Take a leave of absence from your job to provide care for } \\
\text { a loved one }\end{array}$ & 20.9 & 79.1 & 0.0 \\
\hline $\begin{array}{l}\text { c. Go from working full-time to part-time to provide care for } \\
\text { a loved one }\end{array}$ & 15.5 & 84.5 & 0.0 \\
\hline d. Give up working entirely to provide care for a loved one & 21.7 & 74.8 & 3.5 \\
\hline
\end{tabular}




\begin{tabular}{|l|c|c|c|}
\hline & \multicolumn{3}{|c|}{ \% SD39 } \\
\hline $\begin{array}{l}\text { a. Go into work early or late, or take time off to provide care } \\
\text { for a loved one }\end{array}$ & 83.8 & 16.2 & 0.0 \\
\hline $\begin{array}{l}\text { b. Take a leave of absence from your job to provide care for } \\
\text { a loved one }\end{array}$ & 23.8 & 72.2 & 4.0 \\
\hline \begin{tabular}{l} 
c. $\quad \begin{array}{l}\text { Go from working full-time to part-time to provide care for } \\
\text { a loved one }\end{array}$ \\
\hline d. Give up working entirely to provide care for a loved one
\end{tabular} & 19.9 & 78.2 & 1.9 \\
\hline
\end{tabular}

Q9_Q21. You mentioned that you had to go into work early or late, or take time off to provide care to an adult loved one. How often would you say you had to take time away from work or adjust your work schedule to provide care for someone? Would you say ...?

\begin{tabular}{|l|c|c|c|c|}
\hline & \% ALL & \% SD5 & \% SD26 & $\%$ SD39 \\
\hline More than once per week & 12.4 & 11.6 & 2.7 & 16.9 \\
\hline Once per week & 11.2 & 17.6 & 10.0 & 11.7 \\
\hline $\begin{array}{l}\text { Several times per month but less often } \\
\text { than once per week }\end{array}$ & 24.5 & 24.2 & 32.9 & 14.8 \\
\hline Once per month & 24.8 & 22.4 & 11.9 & 34.6 \\
\hline Less than once per month & 21.0 & 13.6 & 37.0 & 14.8 \\
\hline DK/REF & 6.0 & 10.6 & 5.5 & 7.2 \\
\hline
\end{tabular}

Q10_Q22. Now thinking again about the adult loved one you are currently providing/most recently provided care to, did you ever have to ...?

\begin{tabular}{|l|c|c|c|}
\hline & \multicolumn{3}{|c|}{ \% ALL } \\
\hline a. Use your own money to help provide care & Yes & No & NS/REF \\
\hline b. Make changes to your home for the loved one in your care & 63.4 & 34.8 & 1.8 \\
\hline c. Move into another home to accommodate the loved one in & 15.0 & 63.2 & 0.1 \\
\hline & 84.8 & 0.2 \\
\hline
\end{tabular}

\begin{tabular}{|l|c|c|c|}
\hline & \multicolumn{3}{|c|}{$\%$ SD5 } \\
\hline & Yes & No & NS/REF \\
\hline a. Use your own money to help provide care & 54.8 & 41.0 & 4.3 \\
\hline b. Make changes to your home for the loved one in your care & 41.8 & 58.2 & 0.0 \\
\hline $\begin{array}{l}\text { c. } \text { Move into another home to accommodate the loved one in } \\
\text { your care }\end{array}$ & 17.1 & 82.9 & 0.0 \\
\hline
\end{tabular}

\begin{tabular}{|l|c|c|c|}
\hline & \multicolumn{3}{|c|}{$\%$ SD26 } \\
\hline & Yes & No & NS/REF \\
\hline a. Use your own money to help provide care & 63.3 & 35.7 & 1.0 \\
\hline b. Make changes to your home for the loved one in your care & 31.9 & 68.1 & 0.0 \\
\hline $\begin{array}{l}\text { c. Move into another home to accommodate the loved one in } \\
\text { your care }\end{array}$ & 9.2 & 90.8 & 0.0 \\
\hline
\end{tabular}




\begin{tabular}{|c|c|c|c|}
\hline & \multicolumn{3}{|c|}{$\%$ SD39 } \\
\hline & Yes & No & NS/REF \\
\hline a. Use your own money to help provide care & 59.8 & 34.1 & 6.0 \\
\hline b. Make changes to your home for the loved one in your care & 27.5 & 72.5 & 0.0 \\
\hline $\begin{array}{l}\text { c. Move into another home to accommodate the loved one in } \\
\text { your care }\end{array}$ & 22.5 & 77.5 & 0.0 \\
\hline
\end{tabular}

Q11_Q23. As a caregiver do/did you ever ...?

\begin{tabular}{|ll|c|c|c|}
\hline & \multicolumn{3}{|c|}{$\%$ ALL } \\
\hline $\begin{array}{l}\text { a. } \\
\text { Feel stressed emotionally due to your caregiving } \\
\text { responsibilities }\end{array}$ & Yes & No & NS/REF \\
\hline b. Feel stressed in trying to balance your job and family & 65.7 & 32.6 & 0.7 \\
\hline c. & Find it difficult to maintain a healthy diet & 32.1 & 66.9 & 0.5 \\
\hline d. & Find it difficult to get enough rest & 58.0 & 41.9 & 0.1 \\
\hline e. & Find it difficult to exercise regularly & 45.2 & 53.8 & 1.0 \\
\hline f. & Find it difficult to take care of your household \\
& responsibilities & 44.4 & 54.4 & 1.1 \\
\hline g. Experience problems with your health & 30.4 & 69.4 & 0.3 \\
\hline h. Find it difficult to make time to visit your doctor & 21.1 & 78.3 & 0.5 \\
\hline i. $\begin{array}{l}\text { Feel strained financially due to your caregiving } \\
\text { responsibilities }\end{array}$ & 27.4 & 71.9 & 0.7 \\
\hline
\end{tabular}

\begin{tabular}{|c|c|c|c|}
\hline & \multicolumn{3}{|c|}{$\%$ SD5 } \\
\hline & Yes & No & NS/REF \\
\hline $\begin{array}{l}\text { a. Feel stressed emotionally due to your caregiving } \\
\text { responsibilities }\end{array}$ & 71.3 & 27.6 & 1.1 \\
\hline b. Feel stressed in trying to balance your job and family & 68.1 & 31.9 & 0.0 \\
\hline c. Find it difficult to maintain a healthy diet & 37.0 & 63.0 & 0.0 \\
\hline d. Find it difficult to get enough rest & 56.3 & 43.7 & 0.0 \\
\hline e. Find it difficult to exercise regularly & 54.6 & 45.4 & 0.0 \\
\hline $\begin{array}{l}\text { f. Find it difficult to take care of your household } \\
\text { responsibilities }\end{array}$ & 46.2 & 52.8 & 1.0 \\
\hline g. Experience problems with your health & 38.5 & 61.5 & 0.0 \\
\hline h. Find it difficult to make time to visit your doctor & 26.8 & 73.2 & 0.0 \\
\hline $\begin{array}{l}\text { i. Feel strained financially due to your caregiving } \\
\text { responsibilities }\end{array}$ & 32.0 & 67.0 & 1.0 \\
\hline
\end{tabular}




\begin{tabular}{|c|c|c|c|}
\hline & \multicolumn{3}{|c|}{$\%$ SD26 } \\
\hline & Yes & No & NS/REF \\
\hline $\begin{array}{l}\text { a. Feel stressed emotionally due to your caregiving } \\
\text { responsibilities }\end{array}$ & 65.6 & 33.4 & 1.0 \\
\hline b. Feel stressed in trying to balance your job and family & 55.3 & 44.7 & 0.0 \\
\hline c. Find it difficult to maintain a healthy diet & 30.1 & 67.8 & 2.1 \\
\hline d. Find it difficult to get enough rest & 59.1 & 39.9 & 1.0 \\
\hline e. Find it difficult to exercise regularly & 36.3 & 61.6 & 2.1 \\
\hline $\begin{array}{l}\text { f. Find it difficult to take care of your household } \\
\text { responsibilities }\end{array}$ & 43.4 & 55.5 & 1.0 \\
\hline g. Experience problems with your health & 31.4 & 68.6 & 0.0 \\
\hline h. Find it difficult to make time to visit your doctor & 19.0 & $79 . .0$ & 2.0 \\
\hline $\begin{array}{l}\text { i. Feel strained financially due to your caregiving } \\
\text { responsibilities }\end{array}$ & 28.5 & 70.5 & 1.0 \\
\hline
\end{tabular}

\begin{tabular}{|ll|c|c|c|}
\hline & \multicolumn{3}{|c|}{ \% SD39 } \\
\hline & \multicolumn{2}{|c|}{$\begin{array}{c}\text { Yes } \\
\text { a. }\end{array}$} & Noel stressed emotionally due to your caregiving & NS/REF \\
\hline responsibilities & 74.0 & 26.0 & 0.0 \\
\hline b. & Feel stressed in trying to balance your job and family & 66.4 & 31.8 & 1.9 \\
\hline c. & Find it difficult to maintain a healthy diet & 23.3 & 74.3 & 2.4 \\
\hline d. & Find it difficult to get enough rest & 55.8 & 44.2 & 0.0 \\
\hline e. & Find it difficult to exercise regularly & 48.7 & 50.1 & 1.2 \\
\hline f. & Find it difficult to take care of your household \\
& responsibilities & 40.7 & 58.2 & 1.1 \\
\hline g. & Experience problems with your health & 19.2 & 80.8 & 0.0 \\
\hline h. & Find it difficult to make time to visit your doctor & 16.9 & 83.1 & 0.0 \\
\hline i. & Feel strained financially due to your caregiving \\
& responsibilities & 17.6 & 82.4 & 0.0 \\
\hline
\end{tabular}

Q12_Q24. How important is/was it to you to be able to provide care for your loved one so that they can keep living independently in their own home? Is/Was it ...?

\begin{tabular}{|l|c|c|c|c|}
\hline & $\%$ ALL & $\%$ SD5 & \% SD26 & $\%$ SD39 \\
\hline Extremely important & 57.2 & 62.1 & 64.7 & 51.3 \\
\hline Very important & 33.0 & 31.3 & 26.0 & 32.3 \\
\hline Somewhat important & 5.0 & 3.4 & 6.1 & 10.5 \\
\hline Not very important & 1.3 & 0.0 & 1.0 & 1.1 \\
\hline Not at all important & 2.1 & 2.1 & 1.0 & 2.4 \\
\hline DK/REF & 1.4 & 1.0 & 1.0 & 2.4 \\
\hline
\end{tabular}


Q13_Q25. How likely do you think it is that you will provide any caregiving or assistance on an unpaid basis for an adult loved one in the future? Is it ...?

\begin{tabular}{|l|c|c|c|c|}
\hline & \% ALL & \% SD5 & \% SD26 & \% SD39 \\
\hline Extremely likely & 29.6 & 23.6 & 26.6 & 23.9 \\
\hline Very likely & 22.7 & 22.9 & 17.2 & 17.2 \\
\hline Somewhat likely & 16.7 & 14.3 & 23.1 & 23.1 \\
\hline Not very likely & 12.9 & 14.9 & 15.1 & 12.1 \\
\hline Not at all likely & 15.6 & 20.9 & 11.8 & 22.4 \\
\hline NS/REF & 2.5 & 3.4 & 6.2 & 1.3 \\
\hline
\end{tabular}

\section{SKIP TO Q27}

QUESTION 26 IS FOR RESPONDENTS WHO ARE NOT CURRENT OR FORMER CAREGIVERS.

Q26. How likely do you think it is that you will provide any caregiving or assistance on an unpaid basis for an adult loved one in the future? Is it ...?

\begin{tabular}{|l|c|c|c|c|}
\hline & \% ALL & \% SD5 & \% SD26 & \% SD39 \\
\hline Extremely likely & 10.4 & 10.5 & 17.8 & 8.8 \\
\hline Very likely & 14.8 & 14.8 & 7.6 & 24.9 \\
\hline Somewhat likely & 26.3 & 33.5 & 32.4 & 26.8 \\
\hline Not very likely & 23.2 & 22.0 & 18.1 & 19.7 \\
\hline Not at all likely & 22.9 & 16.0 & 24.2 & 17.6 \\
\hline NS/REF & 2.4 & 3.2 & 0.0 & 2.3 \\
\hline
\end{tabular}

Q27. In your opinion, as a current/former/someone who may provide care in the future, how helpful would the following types of caregiving support be to you?

a. Assistance with providing transportation in general.

b. Assistance with providing transportation to medical appointments.

c. Assistance with understanding how to manage medications.

d. Connecting with other caregivers in similar situations.

e. Information about available resources for caregivers in your community.

f. Respite care or breaks from caregiving responsibilities.

g. Assistance with providing meals.

h. Assistance with managing household chores. 


\begin{tabular}{|l|c|c|c|c|c|c|}
\hline & \multicolumn{6}{|c|}{ \% ALL } \\
\hline & $\begin{array}{c}\text { Extremely } \\
\text { Helpful }\end{array}$ & $\begin{array}{c}\text { Very } \\
\text { Helpful }\end{array}$ & $\begin{array}{c}\text { Somewhat } \\
\text { Helpful }\end{array}$ & $\begin{array}{c}\text { Not Very } \\
\text { Helpful }\end{array}$ & $\begin{array}{c}\text { Not at all } \\
\text { Helpful }\end{array}$ & NS/REF \\
\hline a. & 15.4 & 28.7 & 26.7 & 12.9 & 14.9 & 1.3 \\
\hline b. & 17.3 & 28.5 & 24.2 & 12.2 & 16.5 & 1.2 \\
\hline c. & 15.0 & 24.1 & 23.9 & 16.2 & 19.2 & 1.5 \\
\hline d. & 8.6 & 21.3 & 36.2 & 14.5 & 17.2 & 2.2 \\
\hline e. & 19.7 & 33.9 & 25.6 & 8.2 & 10.7 & 2.0 \\
\hline f. & 17.6 & 32.1 & 26.2 & 8.2 & 12.5 & 3.6 \\
\hline g. & 12.4 & 28.8 & 30.2 & 12.0 & 15.0 & 1.5 \\
\hline h. & 13.1 & 29.4 & 28.9 & 13.8 & 13.9 & 1.0 \\
\hline
\end{tabular}

\begin{tabular}{|l|c|c|c|c|c|c|}
\hline & \multicolumn{6}{|c|}{ \% SD5 } \\
\hline & $\begin{array}{c}\text { Extremely } \\
\text { Helpful }\end{array}$ & $\begin{array}{c}\text { Very } \\
\text { Helpful }\end{array}$ & $\begin{array}{c}\text { Somewhat } \\
\text { Helpful }\end{array}$ & $\begin{array}{c}\text { Not Very } \\
\text { Helpful }\end{array}$ & $\begin{array}{c}\text { Not at all } \\
\text { Helpful }\end{array}$ & NS/REF \\
\hline a. & 14.3 & 27.8 & 33.3 & 9.2 & 12.3 & 3.2 \\
\hline b. & 16.2 & 31.3 & 26.0 & 10.6 & 14.8 & 1.2 \\
\hline c. & 18.0 & 22.3 & 28.3 & 16.1 & 14.1 & 1.2 \\
\hline d. & 9.5 & 24.5 & 38.7 & 13.4 & 12.1 & 1.8 \\
\hline e. & 23.0 & 38.8 & 19.0 & 7.3 & 9.8 & 2.0 \\
\hline f. & 22.4 & 32.3 & 26.6 & 5.1 & 8.3 & 5.2 \\
\hline g. & 11.7 & 27.7 & 32.6 & 15.7 & 9.8 & 2.5 \\
\hline h. & 16.1 & 29.5 & 32.4 & 11.8 & 8.4 & 1.9 \\
\hline
\end{tabular}

\begin{tabular}{|l|c|c|c|c|c|c|}
\hline & \multicolumn{6}{|c|}{ \% SD26 } \\
\hline & $\begin{array}{c}\text { Extremely } \\
\text { Helpful }\end{array}$ & $\begin{array}{c}\text { Very } \\
\text { Helpful }\end{array}$ & $\begin{array}{c}\text { Somewhat } \\
\text { Helpful }\end{array}$ & $\begin{array}{c}\text { Not Very } \\
\text { Helpful }\end{array}$ & $\begin{array}{c}\text { Not at all } \\
\text { Helpful }\end{array}$ & NS/REF \\
\hline a. & 16.2 & 21.0 & 32.0 & 15.6 & 14.5 & 0.6 \\
\hline b. & 17.4 & 25.7 & 27.0 & 13.4 & 16.4 & 0.0 \\
\hline c. & 17.2 & 24.3 & 20.7 & 17.7 & 18.2 & 1.9 \\
\hline d. & 10.7 & 17.5 & 34.8 & 16.6 & 16.5 & 3.8 \\
\hline e. & 21.9 & 26.6 & 29.0 & 8.5 & 12.8 & 1.2 \\
\hline f. & 17.2 & 29.6 & 31.7 & 8.8 & 10.8 & 1.9 \\
\hline g. & 14.1 & 29.2 & 26.3 & 12.0 & 16.5 & 1.9 \\
\hline h. & 13.9 & 25.6 & 29.7 & 15.6 & 14.0 & 1.3 \\
\hline
\end{tabular}

\begin{tabular}{|l|c|c|c|c|c|c|}
\hline & \multicolumn{6}{|c|}{ \% SD39 } \\
\hline & $\begin{array}{c}\text { Extremely } \\
\text { Helpful }\end{array}$ & $\begin{array}{c}\text { Very } \\
\text { Helpful }\end{array}$ & $\begin{array}{c}\text { Somewhat } \\
\text { Helpful }\end{array}$ & $\begin{array}{c}\text { Not Very } \\
\text { Helpful }\end{array}$ & $\begin{array}{c}\text { Not at all } \\
\text { Helpful }\end{array}$ & NS/REF \\
\hline a. & 12.5 & 30.6 & 31.6 & 12.2 & 12.5 & 0.6 \\
\hline b. & 10.8 & 31.5 & 28.9 & 11.9 & 15.7 & 1.2 \\
\hline c. & 12.9 & 21.2 & 30.1 & 16.5 & 18.7 & 0.6 \\
\hline d. & 9.2 & 17.4 & 38.6 & 16.0 & 17.3 & 1.6 \\
\hline e. & 19.8 & 35.8 & 29.4 & 5.7 & 6.5 & 2.7 \\
\hline f. & 17.0 & 36.9 & 26.2 & 7.1 & 10.6 & 2.1 \\
\hline g. & 10.4 & 28.5 & 38.0 & 12.0 & 11.2 & 0.0 \\
\hline h. & 10.3 & 27.4 & 31.4 & 14.7 & 16.2 & 0.0 \\
\hline
\end{tabular}


Q28. Family caregivers help older residents in Indiana live independently by performing services like bathing and dressing as well as managing medication and paying bills. How important do you think it is to have more resources and training for family caregivers? Do you think it is?

\begin{tabular}{|l|c|c|c|c|}
\hline & \% ALL & \% SD5 & \% SD26 & \% SD39 \\
\hline Extremely important & 34.2 & 37.9 & 35.1 & 27.3 \\
\hline Very important & 36.3 & 35.2 & 29.6 & 37.7 \\
\hline Somewhat important & 20.7 & 18.0 & 24.8 & 25.8 \\
\hline Not very important & 3.6 & 3.2 & 6.3 & 5.7 \\
\hline Not at all important & 3.6 & 2.5 & 2.9 & 1.9 \\
\hline DK/REF & 1.6 & 3.1 & 1.3 & 1.7 \\
\hline
\end{tabular}

Q29. About how long would it take you, in minutes, to get to ...?

\begin{tabular}{|l|c|c|c|}
\hline & \multicolumn{3}{|c|}{ \% ALL } \\
\hline & The nearest hospital & $\begin{array}{c}\text { A doctor's office for } \\
\text { primary care }\end{array}$ & $\begin{array}{c}\text { A doctor's office for } \\
\text { specialty care }\end{array}$ \\
\hline Less than 5 minutes & 6.3 & 6.2 & 2.8 \\
\hline 5-10 minutes & 28.3 & 26.5 & 16.8 \\
\hline 11-15 minutes & 24.8 & 24.8 & 22.5 \\
\hline 16-30 minutes & 30.2 & 29.8 & 31.0 \\
\hline 31-45 minutes & 6.6 & 7.6 & 11.4 \\
\hline 46 minutes to 1 hour & 1.9 & 2.1 & 7.3 \\
\hline More than 1 hour & 1.2 & 1.6 & 4.8 \\
\hline DK/REF & 0.6 & 1.3 & 3.4 \\
\hline
\end{tabular}

\begin{tabular}{|l|c|c|c|}
\hline & \multicolumn{3}{|c|}{ \% SD5 } \\
\hline The nearest hospital & $\begin{array}{c}\text { A doctor's office for } \\
\text { primary care }\end{array}$ & $\begin{array}{c}\text { A doctor's office for } \\
\text { specialty care }\end{array}$ \\
\hline 5-10 minutes & 3.1 & 6.1 & 2.4 \\
\hline 11-15 minutes & 17.0 & 25.1 & 13.6 \\
\hline 16-30 minutes & 19.7 & 23.2 & 18.1 \\
\hline $31-45$ minutes & 36.7 & 27.6 & 27.5 \\
\hline 46 minutes to 1 hour & 15.0 & 8.7 & 11.0 \\
\hline More than 1 hour & 4.1 & 4.1 & 14.1 \\
\hline DK/REF & 2.6 & 3.2 & 6.1 \\
\hline
\end{tabular}




\begin{tabular}{|l|c|c|c|}
\hline & \multicolumn{3}{|c|}{ \% SD26 } \\
\hline The nearest hospital & $\begin{array}{c}\text { A doctor's office for } \\
\text { primary care }\end{array}$ & $\begin{array}{c}\text { A doctor's office for } \\
\text { specialty care }\end{array}$ \\
\hline Less than 5 minutes & 6.2 & 8.0 & 1.9 \\
\hline $5-10$ minutes & 24.5 & 15.4 & 12.9 \\
\hline 11-15 minutes & 23.5 & 24.4 & 20.0 \\
\hline 16-30 minutes & 37.7 & 41.8 & 37.4 \\
\hline 31-45 minutes & 5.7 & 7.0 & 10.7 \\
\hline 46 minutes to 1 hour & 1.6 & 2.2 & 10.2 \\
\hline More than 1 hour & 0.6 & 1.3 & 5.1 \\
\hline DK/REF & 0.0 & 0.0 & 1.9 \\
\hline
\end{tabular}

\begin{tabular}{|l|c|c|c|}
\hline & \multicolumn{3}{|c|}{ \% SD39 } \\
\hline The nearest hospital & $\begin{array}{c}\text { A doctor's office for } \\
\text { primary care }\end{array}$ & $\begin{array}{c}\text { A doctor's office for } \\
\text { specialty care }\end{array}$ \\
\hline Less than 5 minutes & 10.5 & 7.2 & 5.0 \\
\hline 5-10 minutes & 41.1 & 37.8 & 26.9 \\
\hline 11-15 minutes & 32.8 & 28.3 & 33.5 \\
\hline 16-30 minutes & 12.7 & 18.9 & 24.8 \\
\hline 31-45 minutes & 2.3 & 4.2 & 2.9 \\
\hline 46 minutes to 1 hour & 0.0 & 1.8 & 1.9 \\
\hline More than 1 hour & 0.6 & 0.0 & 0.0 \\
\hline DK/REF & 0.0 & 1.9 & 5.0 \\
\hline
\end{tabular}

Q30. Thinking about the county you live in do you need to travel out of your county to get to...?

\begin{tabular}{|c|c|c|c|}
\hline & \multicolumn{3}{|c|}{$\%$ ALL } \\
\hline & Yes & No & NS/REF \\
\hline a. The nearest hospital & 17.0 & 82.3 & 0.7 \\
\hline b. A doctor's office for primary care & 19.1 & 80.0 & 1.0 \\
\hline $\begin{array}{l}\text { c. A doctor's office for specialty care, like a cardiologist, } \\
\text { neurologist, or other specialist }\end{array}$ & 34.5 & 63.9 & 1.5 \\
\hline
\end{tabular}

\begin{tabular}{|l|c|c|c|}
\hline & \multicolumn{3}{|c|}{$\%$ SD5 } \\
\hline & Yes & No & NS/REF \\
\hline a. The nearest hospital & 33.5 & 65.2 & 1.3 \\
\hline b. A doctor's office for primary care & 28.2 & 69.1 & 2.7 \\
\hline c. A doctor's office for specialty care, like a cardiologist, & 47.0 & 50.9 & 2.1 \\
\hline & & & \\
\hline
\end{tabular}

\begin{tabular}{|l|c|c|c|}
\hline & \multicolumn{3}{|c|}{$\%$ SD26 } \\
\hline & Yes & No & NS/REF \\
\hline a. The nearest hospital & 13.0 & 86.4 & 0.6 \\
\hline b. A doctor's office for primary care & 15.0 & 84.4 & 0.6 \\
\hline $\begin{array}{l}\text { c. A doctor's office for specialty care, like a cardiologist, } \\
\text { neurologist, or other specialist }\end{array}$ & 33.9 & 63.6 & 2.5 \\
\hline
\end{tabular}




\begin{tabular}{|l|c|c|c|}
\hline & \multicolumn{3}{|c|}{ \% SD39 } \\
\hline & Yes & No & NS/REF \\
\hline a. The nearest hospital & 5.2 & 94.8 & 0.0 \\
\hline b. A doctor's office for primary care & 9.9 & 89.5 & 0.6 \\
\hline $\begin{array}{l}\text { c. A doctor's office for specialty care, like a cardiologist, } \\
\text { neurologist, or other specialist }\end{array}$ & 11.5 & 88.0 & 0.5 \\
\hline
\end{tabular}

\section{WORKPLACE FLEXIBILITY}

Q31. Now I'm going to ask if you support or oppose some proposals that would provide help for working family caregivers. Do you support or oppose?

\begin{tabular}{|ll|c|c|c|c|}
\hline & \multicolumn{4}{|c|}{$\%$ ALL } \\
\hline \begin{tabular}{l} 
a. \\
\hline
\end{tabular} & Requiring employers to provide a limited \\
$\begin{array}{l}\text { amount of unpaid leave to employees who } \\
\text { have to take time off for family } \\
\text { caregiving purposes. }\end{array}$ & 82.1 & 13.1 & 2.6 & 2.1 \\
\hline $\begin{array}{l}\text { b. } \\
\begin{array}{l}\text { Requiring employers to provide some } \\
\text { paid leave to all employees that can be } \\
\text { used for family caregiving purposes. }\end{array}\end{array}$ & 77.8 & 16.5 & 3.8 & 1.8 \\
\hline
\end{tabular}

Q32. Is that strongly or somewhat?

\begin{tabular}{|l|c|c|c|c|c|c|}
\hline \multicolumn{1}{|c|}{ \% ALL } \\
\hline & $\begin{array}{c}\text { Strongly } \\
\text { Support }\end{array}$ & $\begin{array}{c}\text { Somewhat } \\
\text { Support }\end{array}$ & Neither & $\begin{array}{c}\text { Somewhat } \\
\text { Oppose }\end{array}$ & $\begin{array}{c}\text { Strongly } \\
\text { Oppose }\end{array}$ & NS/REF \\
\hline a. & 54.1 & 28.0 & 2.6 & 6.2 & 6.9 & 2.1 \\
\hline b. & 48.5 & 29.4 & 3.8 & 9.7 & 6.8 & 1.8 \\
\hline
\end{tabular}

Q31. Now I'm going to ask if you support or oppose some proposals that would provide help for working family caregivers. Do you support or oppose?

\begin{tabular}{|c|c|c|c|c|}
\hline & \multicolumn{4}{|c|}{$\%$ SD5 } \\
\hline & Support & Oppose & Neither & NS/REF \\
\hline $\begin{array}{l}\text { a. Requiring employers to provide a limited } \\
\text { amount of unpaid leave to employees who } \\
\text { have to take time off for family } \\
\text { caregiving purposes. }\end{array}$ & 85.2 & 10.1 & 2.6 & 2.0 \\
\hline $\begin{array}{l}\text { b. Requiring employers to provide some } \\
\text { paid leave to all employees that can be } \\
\text { used for family caregiving purposes. }\end{array}$ & 75.8 & 17.9 & 4.5 & 1.9 \\
\hline
\end{tabular}


Q32. Is that strongly or somewhat?

\begin{tabular}{|l|c|c|c|c|c|c|}
\hline \multicolumn{7}{|c|}{$\%$ SD5 } \\
\hline & $\begin{array}{c}\text { Strongly } \\
\text { Support }\end{array}$ & $\begin{array}{c}\text { Somewhat } \\
\text { Support }\end{array}$ & Neither & $\begin{array}{c}\text { Somewhat } \\
\text { Oppose }\end{array}$ & $\begin{array}{c}\text { Strongly } \\
\text { Oppose }\end{array}$ & NS/REF \\
\hline a. & 57.9 & 27.4 & 2.6 & 5.0 & 5.1 & 2.0 \\
\hline b. & 47.5 & 28.3 & 4.5 & 10.3 & 7.6 & 1.9 \\
\hline
\end{tabular}

Q31. Now I'm going to ask if you support or oppose some proposals that would provide help for working family caregivers. Do you support or oppose?

\begin{tabular}{|c|c|c|c|c|}
\hline & \multicolumn{4}{|c|}{$\%$ SD26 } \\
\hline & Support & Oppose & Neither & NS/REF \\
\hline $\begin{array}{l}\text { a. Requiring employers to provide a limited } \\
\text { amount of unpaid leave to employees who } \\
\text { have to take time off for family } \\
\text { caregiving purposes. }\end{array}$ & 77.2 & 15.9 & 3.2 & 3.7 \\
\hline $\begin{array}{l}\text { b. Requiring employers to provide some } \\
\text { paid leave to all employees that can be } \\
\text { used for family caregiving purposes. }\end{array}$ & 75.6 & 16.5 & 3.8 & 3.2 \\
\hline
\end{tabular}

Q32. Is that strongly or somewhat?

\begin{tabular}{|l|c|c|c|c|c|c|}
\hline \multicolumn{7}{|c|}{$\%$ SD26 } \\
\hline & $\begin{array}{c}\text { Strongly } \\
\text { Support }\end{array}$ & $\begin{array}{c}\text { Somewhat } \\
\text { Support }\end{array}$ & Neither & $\begin{array}{c}\text { Somewhat } \\
\text { Oppose }\end{array}$ & $\begin{array}{c}\text { Strongly } \\
\text { Oppose }\end{array}$ & NS/REF \\
\hline a. & 51.7 & 25.5 & 3.2 & 5.7 & 10.3 & 3.7 \\
\hline b. & 44.1 & 32.4 & 3.8 & 7.1 & 9.4 & 3.2 \\
\hline
\end{tabular}

Q31. Now I'm going to ask if you support or oppose some proposals that would provide help for working family caregivers. Do you support or oppose?

\begin{tabular}{|ll|c|c|c|c|}
\hline & \multicolumn{4}{|c|}{$\%$ SD39 } \\
\hline \begin{tabular}{l} 
a. \\
\hline
\end{tabular} & Requiring employers to provide a limited \\
$\begin{array}{l}\text { amount of unpaid leave to employees who } \\
\text { have to take time off for family } \\
\text { caregiving purposes. }\end{array}$ & 75.2 & 18.6 & 5.0 & 1.1 \\
\hline $\begin{array}{l}\text { b. } \\
\begin{array}{l}\text { Requiring employers to provide some } \\
\text { paid leave to all employees that can be } \\
\text { used for family caregiving purposes. }\end{array}\end{array}$ & 70.6 & 23.3 & 5.5 & 0.6 \\
\hline
\end{tabular}

Q32. Is that strongly or somewhat?

\begin{tabular}{|c|c|c|c|c|c|c|}
\hline \multicolumn{7}{|c|}{$\%$ SD39 } \\
\hline & $\begin{array}{l}\text { Strongly } \\
\text { Support }\end{array}$ & $\begin{array}{l}\text { Somewhat } \\
\text { Support }\end{array}$ & Neither & $\begin{array}{l}\text { Somewhat } \\
\text { Oppose }\end{array}$ & $\begin{array}{c}\text { Strongly } \\
\text { Oppose }\end{array}$ & NS/REF \\
\hline a. & 45.1 & 30.1 & 5.0 & 12.0 & 6.7 & 1.1 \\
\hline b. & 43.5 & 27.1 & 5.5 & 15.4 & 8.0 & 0.6 \\
\hline
\end{tabular}




\section{NURSING SCOPE OF PRACTICE}

Q33. Twenty-two states have passed a proposal to modernize outdated rules related to nurse practitioners and advanced practice registered nurses. These nurses, who have additional training in specific areas like elder care, often visit older patients in their homes. By updating the rules, nurse practitioners can serve as the primary or acute care provider for a patient.

Do you support or oppose modernizing outdated rules to allow nurse practitioners, who have advanced training, to serve as the primary or acute care provider for patients?

\begin{tabular}{|l|c|c|c|c|}
\hline & \% ALL & \% SD5 & \% SD26 & \% SD39 \\
\hline Support & 85.9 & 86.3 & 85.6 & 86.1 \\
\hline Oppose & 8.2 & 8.0 & 6.8 & 8.3 \\
\hline DK/REF & 3.6 & 3.8 & 3.8 & 2.3 \\
\hline
\end{tabular}

Q34. Is that strongly or somewhat?

\begin{tabular}{|l|c|c|c|c|}
\hline & \% ALL & \% SD5 & \% SD26 & \% SD39 \\
\hline Strongly support & 60.4 & 60.0 & 56.9 & 66.7 \\
\hline Somewhat support & 25.5 & 26.3 & 28.7 & 19.5 \\
\hline Neither support nor oppose & 2.3 & 1.9 & 3.8 & 3.4 \\
\hline Somewhat oppose & 3.8 & 3.3 & 0.6 & 2.5 \\
\hline Strongly oppose & 4.4 & 4.7 & 6.1 & 5.8 \\
\hline NS/REF & 3.6 & 3.8 & 3.8 & 2.3 \\
\hline
\end{tabular}

\section{NURSE DELEGATION}

Q35. Some states are considering a proposal that would allow registered nurses to give other health care workers under their supervision the authority to perform certain tasks, such as the administration of medication. This can relieve family caregivers from having to perform the task themselves or hiring a registered nurse to do so.

Do you support or oppose allowing registered nurses in Indiana to delegate some of their responsibilities to other health care workers as long as they were trained to do so?

\begin{tabular}{|l|c|c|c|c|}
\hline & \% ALL & \% SD5 & \% SD26 & $\%$ SD39 \\
\hline Support & 82.1 & 83.7 & 84.5 & 76.6 \\
\hline Oppose & 13.6 & 13.1 & 11.6 & 17.6 \\
\hline DK/REF & 2.7 & 1.9 & 3.2 & 3.2 \\
\hline
\end{tabular}


Q36. Is that strongly or somewhat?

\begin{tabular}{|l|c|c|c|c|}
\hline & \% ALL & \% SD5 & \% SD26 & \% SD39 \\
\hline Strongly support & 47.5 & 45.0 & 50.1 & 45.1 \\
\hline Somewhat support & 34.6 & 38.7 & 34.4 & 31.5 \\
\hline Neither support nor oppose & 1.6 & 1.3 & 0.6 & 2.5 \\
\hline Somewhat oppose & 6.1 & 5.9 & 3.8 & 9.4 \\
\hline Strongly oppose & 7.5 & 7.2 & 7.8 & 8.2 \\
\hline NS/REF & 2.7 & 1.9 & 3.2 & 3.2 \\
\hline
\end{tabular}

\section{INDEPENDENT LIVING}

Q37. If you or a loved one needed help when the basic tasks of life become more difficult due to aging or illness, where would you prefer to receive that care or help for yourself or a loved one?

\begin{tabular}{|l|c|c|c|c|}
\hline & \% ALL & \% SD5 & \% SD26 & \% SD39 \\
\hline a. At home with caregiver assistance & 80.8 & 81.0 & 89.3 & 78.3 \\
\hline $\begin{array}{l}\text { b. } \quad \begin{array}{l}\text { In an assisted living facility or group } \\
\text { home }\end{array} \\
\text { c. } \text { In a nursing home }\end{array}$ & 12.7 & 16.5 & 5.8 & 17.2 \\
\hline d. NS/REF & 3.1 & 1.9 & 1.9 & 2.2 \\
\hline
\end{tabular}

Q38. If you or a loved one needed help to remain independent, how important would it be to have services that would allow you or a loved one to live independently at home for as long as possible? Would it be ...?

\begin{tabular}{|l|c|c|c|c|}
\hline & \% ALL & \% SD5 & \% SD26 & \% SD39 \\
\hline Extremely important & 65.5 & 64.9 & 68.1 & 59.3 \\
\hline Very important & 25.7 & 28.4 & 25.6 & 25.7 \\
\hline Somewhat important & 6.6 & 4.6 & 5.0 & 13.1 \\
\hline Not very important & 0.8 & 1.5 & 0.0 & 0.6 \\
\hline Not at all important & 0.7 & 0.0 & 1.2 & 0.6 \\
\hline DK/REF & 0.7 & 0.6 & 0.0 & 0.6 \\
\hline
\end{tabular}

Q39. How important is it that services that help older adults live independently, such as home health care, personal care, day programs, and services so caregivers can take a break from their caregiving duties, are available in your community? Is it ...?

\begin{tabular}{|l|c|c|c|c|}
\hline & \% ALL & \% SD5 & \% SD26 & \% SD39 \\
\hline Extremely important & 44.9 & 49.8 & 49.4 & 44.0 \\
\hline Very important & 31.4 & 26.8 & 25.7 & 29.1 \\
\hline Somewhat important & 20.1 & 18.7 & 22.1 & 23.7 \\
\hline Not very important & 1.4 & 2.1 & 2.2 & 1.1 \\
\hline Not at all important & 0.9 & 0.6 & 0.6 & 1.6 \\
\hline DK/REF & 1.3 & 1.9 & 0.0 & 0.6 \\
\hline
\end{tabular}


Q40. If you needed information about some aspect of helping take care of a loved one, where would you turn? Would you turn to ... ?

\begin{tabular}{|l|c|c|c|}
\hline & \multicolumn{3}{|c|}{ \% ALL } \\
\hline & Yes & No & NS/REF \\
\hline a. A doctor, nurse, or other health professional & 96.0 & 2.6 & 1.4 \\
\hline b. A social worker or case worker & 62.0 & 32.9 & 5.0 \\
\hline c. A hospital or clinic & 79.3 & 17.7 & 2.9 \\
\hline d. The internet & 54.5 & 42.5 & 3.0 \\
\hline e. Books, magazines, or the library & 43.2 & 53.8 & 3.0 \\
\hline f. The government & 28.5 & 67.0 & 4.5 \\
\hline g. Family or friends & 84.2 & 13.6 & 2.2 \\
\hline h. Your employer & 20.9 & 74.6 & 4.5 \\
\hline i. A religious organization & 49.8 & 46.6 & 3.6 \\
\hline j. An area aging organization & 62.6 & 29.9 & 7.5 \\
\hline k. INConnect Alliance & 13.4 & 40.4 & 46.2 \\
\hline l. Other [specific names of IN AAA 1-16 based on & 42.4 & 35.5 & 22.1 \\
& & & \\
\hline
\end{tabular}

\begin{tabular}{|l|c|c|c|}
\hline & \multicolumn{3}{|c|}{ \% SD5 } \\
\hline & Yes & No & NS/REF \\
\hline a. A doctor, nurse, or other health professional & 95.6 & 1.9 & 2.5 \\
\hline b. A social worker or case worker & 66.2 & 30.0 & 3.8 \\
\hline c. A hospital or clinic & 77.9 & 18.9 & 3.1 \\
\hline d. The internet & 58.3 & 41.1 & 0.6 \\
\hline e. Books, magazines, or the library & 42.3 & 55.8 & 1.9 \\
\hline f. The government & 33.2 & 64.2 & 2.6 \\
\hline g. Family or friends & 84.7 & 14.7 & 0.6 \\
\hline h. Your employer & 16.2 & 80.5 & 3.2 \\
\hline i. A religious organization & 46.8 & 49.4 & 3.8 \\
\hline j. An area aging organization & 61.9 & 32.4 & 5.7 \\
\hline k. INConnect Alliance & 17.0 & 40.5 & 42.5 \\
\hline l. Other [specific names of IN AAA 1-16 based on & 23.0. & 47.9 & 29.1 \\
& & & \\
\hline
\end{tabular}




\begin{tabular}{|c|c|c|c|}
\hline & \multicolumn{3}{|c|}{$\%$ SD26 } \\
\hline & Yes & No & NS/REF \\
\hline a. A doctor, nurse, or other health professional & 96.8 & 1.3 & 1.9 \\
\hline b. A social worker or case worker & 56.8 & 37.8 & 5.3 \\
\hline c. A hospital or clinic & 79.0 & 19.7 & 1.3 \\
\hline d. The internet & 56.3 & 40.6 & 3.1 \\
\hline e. Books, magazines, or the library & 42.6 & 52.4 & 5.0 \\
\hline f. The government & 25.0 & 68.8 & 6.0 \\
\hline g. Family or friends & 82.5 & 15.3 & 2.2 \\
\hline h. Your employer & 22.5 & 72.7 & 4.8 \\
\hline i. A religious organization & 53.1 & 43.8 & 3.1 \\
\hline j. An area aging organization & 61.8 & 30.4 & 7.8 \\
\hline k. INConnect Alliance & 12.5 & 37.0 & 50.5 \\
\hline $\begin{array}{l}\text { 1. Other [specific names of IN AAA 1-16 based on } \\
\text { county] }\end{array}$ & 49.7 & 30.3 & 20.0 \\
\hline
\end{tabular}

\begin{tabular}{|c|c|c|c|}
\hline & \multicolumn{3}{|c|}{$\%$ SD39 } \\
\hline & Yes & No & NS/REF \\
\hline a. A doctor, nurse, or other health professional & 97.5 & 2.5 & 0.0 \\
\hline b. A social worker or case worker & 63.8 & 35.1 & 1.1 \\
\hline c. A hospital or clinic & 76.7 & 21.6 & 1.7 \\
\hline d. The internet & 70.2 & 29.2 & 0.6 \\
\hline e. Books, magazines, or the library & 42.6 & 55.0 & 2.3 \\
\hline f. The government & 23.7 & 74.0 & 2.3 \\
\hline g. Family or friends & 88.5 & 10.3 & 1.2 \\
\hline h. Your employer & 14.9 & 82.8 & 2.4 \\
\hline i. A religious organization & 52.4 & 45.3 & 2.3 \\
\hline j. An area aging organization & 69.9 & 24.7 & 5.5 \\
\hline k. INConnect Alliance & 11.2 & 42.7 & 46.2 \\
\hline $\begin{array}{l}\text { 1. Other [specific names of IN AAA 1-16 based on } \\
\text { county] }\end{array}$ & 33.1 & 43.6 & 23.2 \\
\hline
\end{tabular}

Q41. How familiar are you with INConnect Alliance?

\begin{tabular}{|l|c|c|c|c|}
\hline & \% ALL & \% SD5 & \% SD26 & \% SD39 \\
\hline Very familiar & 0.7 & 0.0 & 0.0 & 0.6 \\
\hline Somewhat familiar & 3.0 & 2.9 & 5.0 & 3.0 \\
\hline Not very familiar & 9.4 & 8.9 & 6.3 & 5.2 \\
\hline Not at all familiar & 85.4 & 87.5 & 86.8 & 90.0 \\
\hline NS/REF & 1.5 & 0.6 & 1.9 & 1.1 \\
\hline
\end{tabular}


Q42. How familiar are you with [INSERT Name of local AAA 1-16]?

\begin{tabular}{|l|c|c|c|c|}
\hline & \% ALL & \% SD5 & \% SD26 & \% SD39 \\
\hline Very familiar & 10.5 & 2.3 & 10.6 & 8.0 \\
\hline Somewhat familiar & 20.9 & 9.0 & 31.4 & 12.7 \\
\hline Not very familiar & 14.2 & 11.5 & 22.2 & 9.3 \\
\hline Not at all familiar & 56.4 & 76.5 & 35.2 & 69.5 \\
\hline NS/REF & 0.9 & 0.6 & 0.6 & 0.6 \\
\hline
\end{tabular}

Q43. How important is it to you to have access to a free service that will help you navigate the complex health care system including care at-home and in the community, and provide information and resources?

\begin{tabular}{|l|c|c|c|c|}
\hline & \% ALL & \% SD5 & \% SD26 & \% SD39 \\
\hline Extremely important & 31.3 & 31.0 & 36.0 & 23.1 \\
\hline Very important & 32.9 & 30.1 & 30.5 & 36.3 \\
\hline Somewhat important & 25.0 & 28.9 & 19.9 & 33.5 \\
\hline Not very important & 4.9 & 3.7 & 5.2 & 2.9 \\
\hline Not at all important & 4.8 & 4.4 & 7.1 & 4.2 \\
\hline DK/REF & 1.2 & 1.9 & 1.2 & 0.0 \\
\hline
\end{tabular}

\section{Demographics}

The following questions are for classification purposes only and will be kept entirely confidential.

D1. What is your current marital status? Are you ...?

\begin{tabular}{|l|c|c|c|c|}
\hline & \% ALL & \% SD5 & \% SD26 & \% SD39 \\
\hline Married & 61.3 & 58.5 & 57.2 & 68.2 \\
\hline $\begin{array}{l}\text { Not married, living with your partner, or } \\
\text { significant other }\end{array}$ & 3.4 & 2.8 & 3.4 & 5.6 \\
\hline Separated & 1.1 & 1.5 & 0.6 & 0.6 \\
\hline Divorced & 10.0 & 10.7 & 10.0 & 7.2 \\
\hline Widowed & 12.4 & 15.0 & 14.6 & 9.8 \\
\hline Currently single, never married & 9.5 & 8.9 & 110.7 & 6.9 \\
\hline NS/REF & 2.2 & 2.6 & 3.5 & 1.7 \\
\hline
\end{tabular}

D2. [IF D1 = 1 ASK: “Are you or your spouse currently a member of A-A-R-P?" IF D1 = 2 ASK: "Are you or your partner currently a member of A-A-R-P?" OTHERWISE ASK "Are you currently a member of A-A-R-P?"]

\begin{tabular}{|l|c|c|c|c|}
\hline & \% ALL & \% SD5 & \% SD26 & \% SD39 \\
\hline $\begin{array}{l}\text { Are you or your spouse currently a } \\
\text { member of AARP? }\end{array}$ & 61.3 & 58.5 & 57.2 & 68.2 \\
\hline $\begin{array}{l}\text { Are you or your partner currently a } \\
\text { member of AARP? }\end{array}$ & 3.4 & 2.8 & 3.4 & 5.6 \\
\hline Are you currently a member of AARP? & 35.2 & 38.7 & 39.4 & 26.2 \\
\hline
\end{tabular}


D3. In general, when compared to most people your age, how would you rate your health? Is it ...?

\begin{tabular}{|l|c|c|c|c|}
\hline & \% ALL & \% SD5 & \% SD26 & \% SD39 \\
\hline Excellent & 19.6 & 19.6 & 19.1 & 35.4 \\
\hline Very good & 35.4 & 36.4 & 26.2 & 34.0 \\
\hline Good & 25.8 & 19.7 & 35.2 & 25.1 \\
\hline Fair & 11.4 & 14.5 & 12.1 & 3.0 \\
\hline Poor & 6.3 & 8.5 & 6.7 & 1.9 \\
\hline NS/REF & 1.5 & 1.3 & 0.6 & 0.6 \\
\hline
\end{tabular}

D4. Thinking about who lives in your household, do you currently ...?

\begin{tabular}{|l|c|c|c|}
\hline & \multicolumn{3}{|c|}{$\%$ ALL } \\
\hline & Yes & No & NS/REF \\
\hline a. Have any children 18 and under living with you & 17.1 & 80.8 & 2.1 \\
\hline b. Have any children 19 and over living with you & 16.8 & 81.2 & 2.0 \\
\hline c. Have any children away at college & 10.5 & 87.8 & 1.7 \\
\hline d. Have a parent or adult relative living with you & 10.7 & 87.5 & 1.9 \\
\hline
\end{tabular}

\begin{tabular}{|l|c|c|c|}
\hline & \multicolumn{3}{|c|}{ \% SD5 } \\
\hline a. Have any children 18 and under living with you & Yes & No & NS/REF \\
\hline b. Have any children 19 and over living with you & 16.8 & 81.2 & 2.0 \\
\hline c. Have any children away at college & 13.2 & 84.1 & 2.6 \\
\hline d. Have a parent or adult relative living with you & 8.7 & 89.3 & 2.0 \\
\hline
\end{tabular}

\begin{tabular}{|l|c|c|c|}
\hline & \multicolumn{3}{|c|}{ \% SD26 } \\
\hline & Yes & No & NS/REF \\
\hline a. Have any children 18 and under living with you & 16.8 & 81.0 & 2.2 \\
\hline b. Have any children 19 and over living with you & 14.3 & 84.5 & 1.3 \\
\hline c. Have any children away at college & 4.3 & 94.1 & 1.6 \\
\hline d. Have a parent or adult relative living with you & 8.4 & 90.0 & 1.6 \\
\hline
\end{tabular}

\begin{tabular}{|ll|c|c|}
\hline & \multicolumn{3}{|c|}{ \% SD39 } \\
\hline a. Have any children 18 and under living with you & Yes & No & NS/REF \\
\hline b. Have any children 19 and over living with you & 18.9 & 79.4 & 1.8 \\
\hline c. Have any children away at college & 15.2 & 83.0 & 1.8 \\
\hline d. Have a parent or adult relative living with you & 17.2 & $81 . .1$ & 1.8 \\
\hline
\end{tabular}


D5. Do you own or rent your primary residence?

\begin{tabular}{|l|c|c|c|c|}
\hline & \% ALL & \% SD5 & \% SD26 & \% SD39 \\
\hline Own & 82.9 & 80.1 & 83.5 & 83.3 \\
\hline Rent & 12.5 & 14.1 & 13.6 & 13.8 \\
\hline Other & 1.6 & 1.3 & 1.2 & 0.6 \\
\hline NS/REF & 3.1 & 4.6 & 2.3 & 2.3 \\
\hline
\end{tabular}

D6. What type of home is your primary residence? Is it ...?

\begin{tabular}{|l|c|c|c|c|}
\hline & \% ALL & \% SD5 & \% SD26 & \% SD39 \\
\hline Single family home & 84.0 & 82.8 & 89.3 & 79.6 \\
\hline Mobile home & 2.8 & 3.1 & 3.1 & 0.0 \\
\hline Town home or duplex & 2.5 & 1.9 & 2.5 & 1.1 \\
\hline Apartment & 4.9 & 5.6 & 1.3 & 7.3 \\
\hline Condominium or Co-op & 2.4 & 3.2 & 0.6 & 7.3 \\
\hline Something else & 0.9 & 1.3 & 1.3 & 2.3 \\
\hline NS/REF & 2.6 & 2.0 & 1.9 & 2.3 \\
\hline
\end{tabular}

D7. What is the highest level of education that you completed? Is it ...?

\begin{tabular}{|l|c|c|c|c|}
\hline & \% ALL & \% SD5 & \% SD26 & \% SD39 \\
\hline 0 to $12^{\text {th }}$ grade, but with no diploma & 5.3 & 3.3 & 6.7 & 0.0 \\
\hline High school graduate or equivalent & 25.4 & 29.1 & 30.8 & 10.0 \\
\hline $\begin{array}{l}\text { Post high school education, but with no } \\
\text { degree }\end{array}$ & 12.2 & 15.0 & 10.6 & 7.4 \\
\hline 2-year degree & 13.1 & 16.2 & 13.9 & 12.1 \\
\hline 4-year degree & 17.3 & 11.5 & 12.4 & 29.0 \\
\hline Post-graduate study, but with no degree & 6.1 & 4.6 & 6.9 & 4.7 \\
\hline Graduate or professional degree & 18.1 & 18.2 & 15.4 & 34.5 \\
\hline NS/REF & 2.6 & 2.0 & 3.3 & 2.4 \\
\hline
\end{tabular}

D8. Which of the following best describes your current employment status? Are you ...?

\begin{tabular}{|l|c|c|c|c|}
\hline & \% ALL & \% SD5 & \% SD26 & \% SD39 \\
\hline Self-employed full-time & 6.2 & 8.3 & 3.8 & 7.5 \\
\hline Self-employed part-time & 3.1 & 4.3 & 2.5 & 4.7 \\
\hline Employed full-time & 29.6 & 21.5 & 24.5 & 33.6 \\
\hline Employed part-time & 6.8 & 9.5 & 6.3 & 9.4 \\
\hline Retired and not working at all & 40.3 & 40.2 & 46.5 & 35.3 \\
\hline Unemployed and looking for work & 2.4 & 2.3 & 0.6 & 2.3 \\
\hline Not in the labor force for other reasons & $8 . .9$ & 11.9 & 14.5 & 4.9 \\
\hline NS/REF & 2.7 & 2.0 & 1.2 & 2.3 \\
\hline
\end{tabular}


D9. Are you of Hispanic, Spanish, or Latino origin or descent?

\begin{tabular}{|l|c|c|c|c|}
\hline & \% ALL & \% SD5 & \% SD26 & \% SD39 \\
\hline Yes & 1.3 & 0.0 & 1.9 & 0.6 \\
\hline No & 95.7 & 97.4 & 94.2 & 97.1 \\
\hline NS/REF & 3.0 & 2.6 & 3.9 & 2.3 \\
\hline
\end{tabular}

D10. What is your race? Are you ...?

\begin{tabular}{|l|c|c|c|c|}
\hline & $\%$ ALL & $\%$ SD5 & \% SD26 & $\%$ SD39 \\
\hline White/Caucasian & 85.8 & 90.2 & 87.0 & 88.6 \\
\hline Black/African-American & 5.3 & 1.3 & 1.9 & 5.2 \\
\hline Native American/Alaskan Native & 0.8 & 0.0 & 1.3 & 0.0 \\
\hline Asian & 0.4 & 1.3 & 0.0 & 0.0 \\
\hline Native Hawaiian/Pacific Islander & 0.1 & 0.0 & 0.6 & 0.0 \\
\hline Some other race & 2.9 & 1.2 & 5.3 & 2.2 \\
\hline NS/REF & 4.6 & 5.9 & 4.0 & 4.1 \\
\hline
\end{tabular}

D11. Thinking about your state elections for Indiana Governor and Legislators in the last 10 years, which of the following best describes your voting behavior? Would you say you vote ...?

\begin{tabular}{|l|c|c|c|c|}
\hline & \% ALL & \% SD5 & \% SD26 & \% SD39 \\
\hline Always & 66.2 & 65.6 & 71.0 & 73.9 \\
\hline Most of the time & 19.8 & 21.6 & 15.6 & 15.3 \\
\hline About half of the time & 5.5 & 4.0 & 4.2 & 4.7 \\
\hline Seldom & 4.1 & 2.1 & 6.7 & 2.7 \\
\hline Never & 1.4 & 2.8 & 0.6 & 1.6 \\
\hline NS/REF & 3.0 & 4.0 & 1.9 & 1.8 \\
\hline
\end{tabular}

D12. Do you consider yourself to be a ...?

\begin{tabular}{|c|c|c|c|c|}
\hline & $\%$ ALL & $\%$ SD5 & \% SD26 & $\%$ SD39 \\
\hline a. Democrat & 26.3 & 31.6 & 18.8 & 19.9 \\
\hline b. Republican & 32.1 & 27.6 & 34.5 & 46.8 \\
\hline c. Independent & 29.4 & 31.6 & 34.9 & 26.5 \\
\hline d. Something else & 3.0 & 1.9 & 4.1 & 0.5 \\
\hline e. NS/REF & 9.2 & 7.3 & 7.6 & 6.2 \\
\hline
\end{tabular}

D13. How would you characterize your political views? Would you say you are?

\begin{tabular}{|ll|c|c|c|c|}
\hline & \% ALL & \% SD5 & \% SD26 & \% SD39 \\
\hline a. & Conservative & 39.3 & 34.9 & 45.3 & 38.4 \\
\hline b. Moderate & 31.8 & 32.0 & 30.8 & 37.4 \\
\hline c. Liberal & 16.4 & 21.2 & 12.1 & 17.4 \\
\hline d. & None of these & 2.2 & 2.1 & 1.8 & 2.2 \\
\hline e. & NS/REF & 10.4 & 9.8 & 10.1 & 4.6 \\
\hline
\end{tabular}


D14. In general, how comfortable are you in using ... Are you ...?
a. The Internet
b. Computers
c. Cell phones or Smart phones

\begin{tabular}{|l|c|c|c|c|c|c|}
\hline & \multicolumn{5}{|c|}{ \% ALL } \\
\hline & $\begin{array}{c}\text { Extremely } \\
\text { Comfortable }\end{array}$ & $\begin{array}{c}\text { Very } \\
\text { Comfortable }\end{array}$ & $\begin{array}{c}\text { Somewhat } \\
\text { Comfortable }\end{array}$ & $\begin{array}{c}\text { Not Very } \\
\text { Comfortable }\end{array}$ & $\begin{array}{c}\text { Not at all } \\
\text { Comfortable }\end{array}$ & NS/REF \\
\hline a. & 26.9 & 26.7 & 22.1 & 6.8 & 12.3 & 5.1 \\
\hline b. & 24.6 & 27.5 & 23.1 & 7.5 & 11.9 & 5.4 \\
\hline c. & 23.6 & 28.2 & 26.2 & 7.5 & 9.6 & 4.8 \\
\hline
\end{tabular}

\begin{tabular}{|l|c|c|c|c|c|c|}
\hline & \multicolumn{6}{|c|}{$\%$ SD5 } \\
\hline & $\begin{array}{c}\text { Extremely } \\
\text { Comfortable }\end{array}$ & $\begin{array}{c}\text { Very } \\
\text { Comfortable }\end{array}$ & $\begin{array}{c}\text { Somewhat } \\
\text { Comfortable }\end{array}$ & $\begin{array}{c}\text { Not Very } \\
\text { Comfortable }\end{array}$ & $\begin{array}{c}\text { Not at all } \\
\text { Comfortable }\end{array}$ & NS/REF \\
\hline a. & 28.1 & 21.2 & 25.0 & 8.7 & 10.7 & 6.4 \\
\hline b. & 26.7 & 19.9 & 27.0 & 9.8 & 9.0 & 7.7 \\
\hline c. & 23.7 & 27.2 & 25.7 & 7.8 & 10.5 & 5.2 \\
\hline
\end{tabular}

\begin{tabular}{|l|c|c|c|c|c|c|}
\hline & \multicolumn{6}{|c|}{ \% SD26 } \\
\hline & $\begin{array}{c}\text { Extremely } \\
\text { Comfortable }\end{array}$ & $\begin{array}{c}\text { Very } \\
\text { Comfortable }\end{array}$ & $\begin{array}{c}\text { Somewhat } \\
\text { Comfortable }\end{array}$ & $\begin{array}{c}\text { Not Very } \\
\text { Comfortable }\end{array}$ & $\begin{array}{c}\text { Not at all } \\
\text { Comfortable }\end{array}$ & NS/REF \\
\hline a. & 22.2 & 23.1 & 29.4 & 10.1 & 10.6 & 4.5 \\
\hline b. & 19.2 & 26.5 & 31.1 & 8.8 & 10.6 & 3.8 \\
\hline c. & 19.5 & 29.7 & 32.1 & 6.9 & 7.5 & 4.5 \\
\hline
\end{tabular}

\begin{tabular}{|l|c|c|c|c|c|c|}
\hline & \multicolumn{6}{|c|}{ \% SD39 } \\
\hline & $\begin{array}{c}\text { Extremely } \\
\text { Comfortable }\end{array}$ & $\begin{array}{c}\text { Very } \\
\text { Comfortable }\end{array}$ & $\begin{array}{c}\text { Somewhat } \\
\text { Comfortable }\end{array}$ & $\begin{array}{c}\text { Not Very } \\
\text { Comfortable }\end{array}$ & $\begin{array}{c}\text { Not at all } \\
\text { Comfortable }\end{array}$ & NS/REF \\
\hline a. & 40.8 & 29.7 & 18.7 & 1.7 & 5.7 & 3.5 \\
\hline b. & 36.7 & 30.6 & 21.9 & 1.7 & 5.2 & 4.0 \\
\hline c. & 35.3 & 29.8 & 23.1 & 2.9 & 5.9 & 2.9 \\
\hline
\end{tabular}

D15. Have you ever used any of the following healthcare technology for yourself or someone else?

\begin{tabular}{|c|c|c|c|}
\hline & \multicolumn{3}{|c|}{$\%$ ALL } \\
\hline & Yes & No & NS/REF \\
\hline $\begin{array}{l}\text { a. Tools that measure and track fitness or activity levels, } \\
\text { like a Fit-Bit or pedometer }\end{array}$ & 32.2 & 62.7 & 5.1 \\
\hline b. Tools that alert you to take medications on time & 9.5 & 86.2 & 4.2 \\
\hline $\begin{array}{l}\text { c. Tools that measure your overall health, like smart } \\
\text { scales or diet and nutrition websites or APPS }\end{array}$ & 27.2 & 67.8 & 4.9 \\
\hline $\begin{array}{l}\text { d. Personal emergency response systems that let you call } \\
\text { for help easily in an emergency }\end{array}$ & 22.0 & 73.8 & 4.2 \\
\hline $\begin{array}{l}\text { e. An online support community for caregivers, like the } \\
\text { A-A-R-P Caregiver Resource Center }\end{array}$ & 6.7 & 88.3 & 5.0 \\
\hline
\end{tabular}




\begin{tabular}{|l|c|c|c|}
\hline & \multicolumn{3}{|c|}{$\%$ SD5 } \\
\hline $\begin{array}{l}\mid \\
\text { a. Tools that measure and track fitness or activity levels, } \\
\text { like a Fit-Bit or pedometer }\end{array}$ & 32.6 & 62.9 & 4.6 \\
\hline b. Tools that alert you to take medications on time & 10.0 & 86.1 & 3.9 \\
\hline $\begin{array}{l}\text { c. } \quad \text { Tools that measure your overall health, like smart } \\
\text { scales or diet and nutrition websites or APPS }\end{array}$ & 29.8 & 65.0 & 5.2 \\
\hline $\begin{array}{l}\text { d. Personal emergency response systems that let you call } \\
\text { for help easily in an emergency }\end{array}$ & 24.7 & 70.8 & 4.5 \\
\hline $\begin{array}{l}\text { e. } \\
\text { An online support community for caregivers, like the } \\
\text { A-A-R-P Caregiver Resource Center }\end{array}$ & 6.3 & 89.2 & 4.5 \\
\hline
\end{tabular}

\begin{tabular}{|c|c|c|c|}
\hline & \multicolumn{3}{|c|}{$\%$ SD26 } \\
\hline & Yes & No & NS/REF \\
\hline $\begin{array}{l}\text { a. Tools that measure and track fitness or activity levels, } \\
\text { like a Fit-Bit or pedometer }\end{array}$ & 23.9 & 69.1 & 7.1 \\
\hline b. Tools that alert you to take medications on time & 11.8 & 81.8 & 6.4 \\
\hline $\begin{array}{l}\text { c. Tools that measure your overall health, like smart } \\
\text { scales or diet and nutrition websites or APPS }\end{array}$ & 20.3 & 72.0 & 7.8 \\
\hline $\begin{array}{l}\text { d. Personal emergency response systems that let you call } \\
\text { for help easily in an emergency }\end{array}$ & 20.5 & 73.0 & 6.5 \\
\hline $\begin{array}{l}\text { e. An online support community for caregivers, like the } \\
\text { A-A-R-P Caregiver Resource Center }\end{array}$ & 5.7 & 86.6 & 7.7 \\
\hline
\end{tabular}

\begin{tabular}{|c|c|c|c|}
\hline & \multicolumn{3}{|c|}{$\%$ SD39 } \\
\hline & Yes & No & NS/REF \\
\hline $\begin{array}{l}\text { a. Tools that measure and track fitness or activity levels, } \\
\text { like a Fit-Bit or pedometer }\end{array}$ & 46.1 & 46.7 & 7.2 \\
\hline b. Tools that alert you to take medications on time & 4.8 & 89.4 & 5.8 \\
\hline $\begin{array}{l}\text { c. Tools that measure your overall health, like smart } \\
\text { scales or diet and nutrition websites or APPS }\end{array}$ & 32.5 & 62.3 & 5.2 \\
\hline $\begin{array}{l}\text { d. Personal emergency response systems that let you call } \\
\text { for help easily in an emergency }\end{array}$ & 18.2 & 76.6 & 5.2 \\
\hline $\begin{array}{l}\text { e. An online support community for caregivers, like the } \\
\text { A-A-R-P Caregiver Resource Center }\end{array}$ & 6.6 & 87.6 & 5.8 \\
\hline
\end{tabular}


D16. We realize income is a private matter and so rather than ask you anything specific about your income, I'd like to ask you to please stop me when I get to the category that includes your household's income before taxes in 2016. Was it ...?

\begin{tabular}{|l|c|c|c|c|}
\hline & \% ALL & \% SD5 & \% SD26 & \% SD39 \\
\hline Less than $\$ 10,000$ & 3.8 & 5.2 & 7.5 & 1.3 \\
\hline$\$ 10,000$ to less than $\$ 20,000$ & 7.2 & 6.8 & 6.1 & 2.3 \\
\hline$\$ 20,000$ to less than $\$ 30,000$ & 9.4 & $9 . .0$ & 11.3 & 4.0 \\
\hline$\$ 30,000$ to less than $\$ 40,000$ & 8.0 & 10.9 & 7.5 & 3.5 \\
\hline$\$ 40,000$ to less than $\$ 50,000$ & 8.4 & 6.8 & 11.4 & 7.0 \\
\hline$\$ 50,000$ to less than $\$ 55,000$ & 8.2 & 8.7 & 10.7 & 2.2 \\
\hline$\$ 55,001$ to less than $\$ 75,000$ & 7.5 & 9.6 & 6.3 & 9.9 \\
\hline$\$ 75,000$ to less than $\$ 100,000$ & 11.0 & 9.9 & 5.3 & 11.4 \\
\hline$\$ 100,000$ to less than $\$ 125,000$ & 7.0 & 6.7 & 6.3 & 8.3 \\
\hline$\$ 125,000$ to less than $\$ 150,000$ & 4.2 & 3.2 & 1.2 & 10.9 \\
\hline$\$ 150,000$ to less than $\$ 200,000$ & 3.0 & 2.1 & 1.7 & 9.3 \\
\hline$\$ 200,000$ and more & 3.7 & 2.7 & 0.6 & 10.7 \\
\hline NS/REF & 18.7 & 18.4 & 23.8 & 19.2 \\
\hline
\end{tabular}

That was our last question for tonight/today. Thank you very much for taking the time to help us out. Have a great day/night! 